\title{
Polyethyleneimine and Chitosan Polymer-Based Mucoadhesive Liquid Crystalline Systems Intended for Buccal Drug Delivery
}

\author{
Giovana Maria Fioramonti Calixto, ${ }^{1,3}$ Francesca Damiani Victorelli, ${ }^{1}$ Lívia Nordi Dovigo, ${ }^{2}$ and Marlus Chorilli ${ }^{1,3}$
}

Received 9 August 2017; accepted 21 September 2017; published online 10 October 2017

\begin{abstract}
The buccal mucosa is accessible, shows rapid repair, has an excellent blood supply, and shows the absence of the first-pass effect, which makes it a very attractive drug delivery route. However, this route has limitations, mainly due to the continuous secretion of saliva ( 0.5 to $2 \mathrm{~L} /$ day), which may lead to dilution, possible ingestion, and unintentional removal of the active drug. Nanotechnology-based drug delivery systems, such as liquid crystalline systems (LCSs), can increase drug permeation through the mucosa and thereby improve drug delivery. This study aimed at developing and characterizing the mechanical, rheological, and mucoadhesive properties of four liquid crystalline precursor systems (LCPSs) composed of four different aqueous phases (i) water (FW), (ii) chitosan (FC), (iii) polyethyleneimine (FP), or (iv) both polymers (FPC); oleic acid was used as the oil phase, and ethoxylated and propoxylated cetyl alcohol was used as the surfactant. Polarized light microscopy and small-angle X-ray scattering indicated that all LCPSs formed liquid crystalline states after incorporation of saliva. Rheological, texture, and mucoadhesive assays showed that FPC had the most suitable characteristics for buccal application. In vitro release study showed that FPC could act as a controlled drug delivery system. Finally, based on in vitro cytotoxicity data, FPC is a safe buccal drug delivery system for the treatment of several buccal diseases.
\end{abstract}

KEY WORDS: drug delivery system; buccal route; liquid crystalline system; chitosan; polyethyleneimine.

\section{INTRODUCTION}

The buccal mucosa is permeable and robust, allowing for rapid recovery after stress or damage. It has an excellent blood supply and has an absence of the first-pass effect, avoiding the drug metabolism which occurs pre-systemically in the gastrointestinal tract. Together, these characteristics make the buccal mucosa a very attractive drug administration route (1). Despite its advantages, buccal administration has some limitations, mainly due to the continuous secretion of saliva ( 0.5 to $2 \mathrm{~L} /$ day), which may lead to dilution and possible ingestion of the drug and, lastly, to the unintentional removal of the pharmaceutical form (2).

Nanostructured release systems encompassing liquid crystalline systems, micro-emulsions, polymer nanoparticles, solid lipid nanoparticles, and nanostructured lipid carriers represent promising platforms for buccal administration of

\footnotetext{
${ }^{1}$ Faculdade de Ciências Farmacêuticas, Departamento de Fármacos e Medicamentos, UNESP-Universidade Estadual Paulista, Campus Araraquara, Araraquara, SP 14800-850, Brazil.

${ }^{2}$ Araraquara Dental School, UNESP- Univ Estadual Paulista, Araraquara, SP 14801-903, Brazil.

${ }^{3}$ To whom correspondence should be addressed. (e-mail: calixtogmf@fcfar.unesp.br; chorilli@fcfar.unesp.br)
}

drugs, since these systems can protect them from degradation and increase the residence time of the formulation in the buccal environment, allowing for controlled drug release at the site of action (3).

Among these systems, lyotropic liquid crystalline systems (LCSs) stand out because they are formed by surfactants that, following the gradual addition of solvents such as water, form lamellar, hexagonal, or cubic crystalline liquid mesophases. The lamellar mesophase is a onedimensional structure formed by parallel and planar bilayers of surfactant separated by layers of solvent. The hexagonal mesophase is formed by layers of surfactant and solvent that are arranged in the form of cylinders forming a two-dimensional structure. Finally, the cubic mesophase is formed by two networks of solvent channels surrounded by bilayers of surfactant arranged in a threedimensional organized structure $(4,5)$. Therefore, the gradual increase in water in the LCS increases the structural organization of the system, which results in an increase of the viscosity of the formulation.

Thus, liquid crystal precursor systems (LCPSs) are of particular interest for the buccal administration of drugs, since LCPSs are liquid, which will facilitate administration of the formulation, for example, by syringe. However, when it comes in contact with the buccal environment, a LCPS has 
the capacity to incorporate water from the saliva, becoming a more viscous liquid crystalline mesophase, which can promote drug-controlled release and result in greater drug targeting in the buccal mucosa $(6,7)$.

The surfactant ethoxylated and propoxylated cetyl alcohol (PPG-5-CETETH-20) stands out as being a nonionic surfactant which can form LCS without the addition of a cosurfactant, due to its capacity for self-organization in the absence of repulsive electrostatic interactions, that favors a low surface tension $(8,9)$. Furthermore, PPG-5-CETETH-20 exhibits pharmaceutical characteristics that encourage its use as excipient for drug delivery system, such as colorless liquid, light odor, completely soluble in water, and ethanol. Furthermore, this surfactant is not skin irritant and its lethal dose 50 in rats is $3050 \mathrm{mg} \mathrm{kg}^{-1}(10,11)$.

In order to prolong drug release and decrease the need for repeated administration of drugs, the contact time of the formulation with the buccal mucosa needs to be optimized, and this can be achieved using mucoadhesive polymer dispersions as the aqueous phase for the LCPS, since these polymers have excellent adhesiveness to the buccal mucosa (12).

Chitosan (CS), $\alpha$ (1-4) -2-amino-2-deoxy $\beta$-D-glucan, a deacetylated form of chitin, is a biocompatible, biodegradable cationic polysaccharide, with low cytotoxicity, that has been successfully employed in several drug release systems $(13,14)$ This polymer has also been widely used to optimize buccal administration of drugs, as it has an excellent mucoadhesive capacity. This high mucoadhesion is caused by molecular attraction through electrostatic interactions with the negatively charged saliva. In addition, QS can act as a promoter of absorption $(1,15)$.

Polyethyleneimines (PEI) are water-soluble polymers that have a high cationic charge density at physiological $\mathrm{pH}$; this is due to the presence of protonatable amino groups at each third position in the molecule (16). PEI has also been studied extensively in cancer therapy with the aim of promoting the cellular uptake of drugs, because this positively charged polymer has the ability to interact with the negatively charged extracellular membranes, which typically contain high levels of the anionic phospholipid phosphatidylserine (14).

One study has reported the association of PEI with QS for the administration of antitumor drug with the aim of increasing affinity for the target cell. In this study, Jere et al. (14) developed a copolymer system composed of PEI and QS that allowed for incorporation of the antitumor drug. The authors optimized treatment of lung tumor cells with this antitumor agent when it was incorporated into the PEI/QS system along with associated polymers (14).

Furthermore, studies by our research group have shown that SLC formed by polymers as aqueous phase can act as bio(muco)adhesive systems in addition to promoting the increase of permeation and retention of the drug by the biological surface $(8,9,17-21)$.

Finally, the addition of an oil phase, such as oleic acid (OA), can also be considered in the SLC composition, because $\mathrm{OA}$ can act as a drug permeation agent. It exhibits a mechanism of interaction with the carbonic chains of the lipids from membrane which leads to a packaging perturbation in the region of the polar heads of the lipid bilayer and then to the fluidity of the membrane phospholipid domains, which results in the greater permeability of the drug $(22,23)$. Considering the advantages that the SLC can offer in the development of drug delivery mucoadhesive systems and the promising results obtained by our research group in relation to the PPG-5-CETETH-20 surfactant with the addition of bioadhesive polymers, this work aims the pharmaco-technical development of novel LCPSs comprising of PPG-5CETETH-20 as the surfactant, oleic acid as the oil phase, and CS/PEI dispersion as the aqueous phase following their characterization by polarized light microscopy (PLM), smallangle X-ray scattering (SAXS), and rheological, mechanical, and bioadhesion assays to explore these technological platforms for the development of a mucoadhesive nanostructured delivery system for buccal administration of drugs.

\section{MATERIALS AND METHODS}

\section{Materials}

PPG-5-CETETH-20 was purchased from Croda (Campinas, Sao Paulo, Brazil). Oleic acid was purchased from Synth (Diadema, Sao Paulo, Brazil). Branched polyethylenimine with molecular weight of $25 \mathrm{kDa}$ and lowmolecular-weight chitosan were purchased from Sigma Aldrich ${ }^{\circledR}$ (Steinheim, North Rhine-Westphalia, Germany). High-purity water was prepared with a Millipore Milli-Q Plus purification system (Merck Millipore Corporation ${ }^{\circledR}$, Darmstadt, Hessen, Germany).

\section{Phase Diagram Construction and Preparation of Liquid Crystalline Systems}

Four different phase diagrams were constructed using oleic acid as the oil phase and PPG-5-CETETH-20 as the surfactant in all four diagrams. Four different aqueous phases were used, namely high-purity water (diagram 1), a dispersion of $0.5 \%$ chitosan (diagram 2), a dispersion of $0.5 \%$ polyethyleneimine (diagram 3 ), and a binary dispersion of $0.25 \%$ polyethyleneimine and $0.25 \%$ chitosan (diagram 4 ). Fifty-four different proportions, varying by $10 \%$ in $10 \%(w /$ $w$ ) for each phase of the systems, were mixed by vortexing at $25 \pm 0.5^{\circ} \mathrm{C}$. For diagrams with $\mathrm{CS}$ or PEI dispersion as the aqueous phase, the dispersion was initially prepared at $5 \%$ PEI or CS $(w / w)$. Then, this dispersion was mixed with different amounts of water, so as to result in $0.5 \%$ polymer percentage in the systems. For diagram with the binary dispersion of $0.25 \%$ polyethyleneimine and $0.25 \%$ chitosan, the binary dispersion was initially prepared at $2.5 \%$ PEI and $2.5 \% \operatorname{CS}(w / w)$. Then, this dispersion was mixed with different amounts of water, so as to result in $0.25 \%$ polymer percentage in the systems. The systems were allowed to stand for $48 \mathrm{~h}$ to allow for complete stabilization and elimination of bubbles. After this time, the systems were visually classified as a transparent liquid system (TLS), a transparent viscous system (TVS), an opaque system (OS), and a phase separation (PS). Using this classification, the phase diagrams were plotted using SigmaPlot $\AA$, version 10.0 (Systat Software, USA).

Then, one of LCPSs from each diagram was selected for the other studies, which were named FW (diagram 1), FC (diagram 2), FP (diagram 3), and FPC (diagram 4). 
To verify if the selected formulations behaved like LCPS, there were added 30\% (FW30, FC30, FP30, FPC30) and 100\% (FW100, FC100, FP100, FPC100) of artificial saliva into FW, FC, FP, and FPC. The composition of the artificial saliva used was $8 \mathrm{~g} / \mathrm{L}$ of sodium chloride $(\mathrm{NaCl}), 0.19 \mathrm{~g} / \mathrm{L}$ of potassium monobasic phosphate $\left(\mathrm{KH}_{2} \mathrm{PO}_{4}\right)$, and $2.28 \mathrm{~g} / \mathrm{L}$ of disodium phosphate $\left(\mathrm{Na}_{2} \mathrm{HPO}_{4}\right)$ with $\mathrm{pH} 6.8$ (24).

All systems were classified visually and by polarized light microscopy and characterized by small-angle X-ray scattering (SAXS), continuous rheological analysis, oscillatory rheological analysis, and mucoadhesion.

\section{Structural Characterization of Selected Formulations}

\section{Polarized Light Microscopy}

A drop of each formulation was placed on a glass slide, covered with a cover slip and examined under polarized light at room temperature $\left(25 \pm 0.5^{\circ} \mathrm{C}\right)$ using an Olympus BX41 polarized light microscope coupled with a Q-Color3 camera (Olympus America Inc.). The magnification was 20×.

\section{Small-Angle X-ray Scattering}

The structures of the formulations were analyzed by SAXS using the Brazilian Synchrotron Light Laboratory instrument (LNLS, Campinas, Brazil) equipped with a type $\mathrm{Si}$ (111) monochromator at a wavelength $(\lambda)$ of $1.608 \AA$ with a horizontally focused beam. A vertical Pilatus 300K SAXS detector located at $858.45 \mathrm{~mm}$ from the sample, and 13 multichannel analyzers were employed to record the intensity of the scattering $I(q)$ from 0.1 to $3.8 \AA^{-1}$ at $25{ }^{\circ} \mathrm{C}$. The scattering observed in the system not containing a sample was subtracted from the total intensity of the sample, as a function of the modulus of the scattering vector $q, q=4 \pi \sin \theta / \lambda$, where $\lambda$ is the wavelength and $2 \theta$ is the scattering angle. The intensities of all samples were measured in relative units, and a quantitative comparison of the measurements was standardized using the same experimental conditions.

\section{Texture Profile Analysis}

Texture profile analysis (TPA) was performed using a TA-XTplus texture analyzer (Stable Micro Systems, Surrey, UK) in the TPA mode. The selected formulations and a commercial buccal formulation ( $8 \mathrm{~g}$ of each sample) were centrifuged at $2000 \mathrm{~g}$ for $10 \mathrm{~min}$ (5810R, Eppendorf, New York, NY, USA), and the cylindrical analytical probe $(10 \mathrm{~mm}$ diameter) was lowered $\left(1 \mathrm{~mm} \mathrm{~s}^{-1}\right)$ until it reached the sample. The formulations were compressed twice $\left(0.5 \mathrm{~mm} \mathrm{~s}^{-1} ; 10-\mathrm{mm}\right.$ depth; 5-s delay period). Hardness, compressibility, adhesiveness, and cohesion were calculated from the force-time curves through the program Expert Texture Exponent. Seven replicates were analyzed at $25 \pm 0.5^{\circ} \mathrm{C}$.

\section{In Vitro Evaluation of the Mucoadhesive Force}

In order to evaluate the in vitro mucoadhesive strength of the selected formulations and a commercial buccal formulation, swine esophageal mucosae, obtained from the Olhos d'Água slaughterhouse in Ipuã (SP), were transported and stored in saliva medium on ice for up to a maximum of $6 \mathrm{~h}$ following death of the animal. The esophageal mucosa was separated from the underlying tissues using anatomical scissors and a scalpel, maintaining a uniform 1-mm-thick cut. The mucosa was washed with running water and stored in saliva until use. The strength required to remove the formulation from the swine esophageal mucosa was evaluated in vitro using a TA-XT plus texture analyzer (Stable Micro Systems, Surrey, England) in the adhesion test mode. First, the mucosa was immersed in human saliva to simulate the buccal environment for $30 \mathrm{~s}$. The mucosa was then attached to the lower end of the cylindrical probe (10-mm diameter). The formulations were then placed in small glass containers under the probe at $37{ }^{\circ} \mathrm{C}$. The test was started by lowering the probe at a constant rate $(1 \mathrm{~mm} / \mathrm{s})$ until the mucosa came in contact with the sample. The mucosa and sample were held in contact for $60 \mathrm{~s}$, during which time no force was applied. Thereafter, the probe rose at a constant rate $(0.5 \mathrm{~mm} / \mathrm{s})$, until detachment of the mucosa from the sample occurred. The force required for this detachment to occur was calculated from the force versus time curve.

\section{Rheological Analysis}

The rheological analysis was performed at $37 \pm 0.1{ }^{\circ} \mathrm{C}$ in triplicate, using a controlled-stress AR2000 rheometer (TA Instruments, New Castle, DE, USA) with a plate diameter of $40 \mathrm{~mm}$ and a sample gap of $200 \mu \mathrm{m}$. Samples of the formulations were carefully applied to the lower plate to minimize sample shearing and were allowed to equilibrate for $3 \mathrm{~min}$ prior to analysis.

\section{Determination of Flow Properties}

The flow properties were determined using a controlled shear rate procedure ranging from 0.01 to $100 \mathrm{~s}^{-1}$ and back. Each stage lasted $120 \mathrm{~s}$ with an interval of $10 \mathrm{~s}$ between the curves. The consistency and flow indexes were determined from the power law described in Eq. 1 for a quantitative analysis of flow behavior:

$$
\tau=k \cdot \gamma^{\eta}
$$

where " $\tau$ " is the shear stress $(\mathrm{Pa})$, " $\gamma$ " is the shear rate $(1 / \mathrm{s})$, " $k$ " is the consistency index $\left[(\mathrm{Pa} \mathrm{s})^{n}\right]$, and " $n$ "(dimensionless) is the flow index.

\section{Oscillatory Analyses}

Oscillatory analyses were started by conducting a stress sweep in order to determine the viscoelastic region of the formulations. The stress sweep was carried out at a constant frequency of $1 \mathrm{~Hz}$, over a stress range of $0.1-10 \mathrm{~Pa}$. A constant shear stress of $1 \mathrm{~Pa}$ was selected to perform the frequency sweep over a range of $0.1-10 \mathrm{~Hz}$, which was within the previously determined linear viscoelastic region for all formulations. The storage $\left(\mathrm{G}^{\prime}\right)$ and loss $\left(\mathrm{G}^{\prime \prime}\right)$ moduli were recorded. 


\section{In Vitro Release Study}

In vitro release of the peptide drug (CTT1) from the formulations FW and FPC was determined using a Franz cell apparatus (Hanson Research Corporation, Chatsworth, CA, USA). Firstly, CTT1 was incorporate at $100 \mu \mathrm{g} / \mathrm{mL}$ into FW (FW-CTT1) and FPC (FPC-CTT1). A synthetic cellulose acetate membrane (molar mass cutoff $12-14 \mathrm{kDa}$ ) with an area of $1.77 \mathrm{~cm}^{2}$ was previously treated with Milli-Q water for $5 \mathrm{~min}$. Samples of the formulations $(300 \mathrm{mg})$ were placed on the membrane surface at the donor compartment. The latter compartment was filled with $7 \mathrm{~mL}$ of $0.2 \mathrm{M}$ phosphate buffer (pH 7.4). The receptor solution was constantly stirred at $300 \mathrm{rpm}$ and maintained at $37.0 \pm 0.5{ }^{\circ} \mathrm{C}$ in sink conditions. The released samples $(1.8 \mathrm{~mL})$ were collected automatically after $5 \mathrm{~min}, 30 \mathrm{~min}, 1 \mathrm{~h}, 2 \mathrm{~h}, 4 \mathrm{~h}, 8 \mathrm{~h}, 12 \mathrm{~h}, 18 \mathrm{~h}$, and $24 \mathrm{~h}$, and were replaced by the same amount of fresh buffer. At the end of the experiment, the amount of CTT1 released from the formulations at each time interval was analyzed by a spectrophotometer at a wavelength of $280 \mathrm{~nm}$ (HP 8453 Agilent, London, UK). The results are expressed as an average of six measurements and the error is reported as standard deviation (SD).

\section{In Vitro Cytotoxicity}

Cell viability tests were performed using the immortalized human keratinocyte line $\mathrm{HaCaT}$ as the cell model. Cells were seeded at a density of $2.5 \times 10^{5}$ to $10 \times 10^{5}$ cells/well (in 96-well plates), cultured for $48 \mathrm{~h}$ in the presence of different doses of the formulation $(1.87-0.06 \mathrm{mg} / \mathrm{mL})$. After treatment, the cells were washed once with PBS, to remove the formulation. Cell viability was assessed using a 3-(4,5dimethylthiazol-2-yl)-2,5-diphenyltetrazolium bromide (MTT) reduction test, which is based on the reduction of MTT to formazan by mitochondrial dehydrogenase. Cells and MTT $(0.4 \mathrm{mg} / \mathrm{mL})$ were incubated in humidified atmosphere at $37{ }^{\circ} \mathrm{C}$ for $3 \mathrm{~h}$. After the incubation period, the supernatant was removed and formazan crystals were dissolved in DMSO $(180 \mu \mathrm{L})$. The plates were shaken for $10 \mathrm{~min}$, and the optical densities were measured at $540 \mathrm{~nm}$ in a multi-well spectrophotometer. Each concentration was assayed three times and six additional controls (cells in medium) were used for each test. Cell viability was calculated using Eq. 2 as follows:

Cell viability $(\%)=[\mathrm{OD} 540($ sample $) / \mathrm{OD} 540($ control $)] \times 100$.

\section{Statistical Analyses}

Statistical analyses were performed using standard methods. ANOVA was employed to calculate significance $(p<0.05)$.

\section{RESULTS AND DISCUSSION}

The ternary phase diagram is a tool used to visualize the required amount of oil phase, aqueous phase, and surfactant required for the formation of different pharmaceutical forms, such as emulsions, micro-emulsions, and liquid crystalline systems. These diagrams are represented by an equilateral triangle where the upper vertex of the triangle represents $100 \%$ surfactant, the left-hand vertex represents $100 \%$ aqueous phase, and the right-hand vertex represents $100 \%$ of the oil phase (25). Therefore, construction of these diagrams serves as a starting point for the study of the design of pharmaceutical forms, since it reveals the physical and chemical behaviors of the mixtures of different proportions of the phases, facilitating the choice of the formulation most suitable for the proposed purpose (26).

The four diagrams that were constructed are shown in Fig. 1 which allows for a visual comparison of the different aqueous phases.

Phase diagram 1 uses PPG-5-CETETH-20 as the surfactant, oleic acid (OA) as the oil phase, and water as the aqueous phase, and is shown in Fig. 1(1)). Close analysis of the diagram reveals that at a concentration below $40 \%$ PPG5-CETETH-20, a large phase separation (PS) region, with a small opaque system (OS) region, was found between 10 and $25 \%$ surfactant phases, and between 20 and $30 \%$ oil phases. A wide region of transparent liquid system (TLS) was obtained below $25 \%$ water, covering almost every axis of the surfactant and the oil phase. From 40 to $75 \%$ surfactant, from 25 to $75 \%$ water, and below $50 \%$ oil, a region of viscous system (VS) was obtained. Similar data has been reported by Carvalho et al. (2013) (27).

Diagram 2 shown in Fig. 1(2)) represents the systems obtained using PPG-5-CETETH-20 as surfactant, OA as the oil phase, and $0.5 \%$ chitosan dispersion (CS) as the aqueous phase. Two small phase separation regions were noted: (i) between 5 and $25 \%$ surfactants, 5 and $35 \%$ aqueous phases, and 45 and $85 \%$ oil phases and (ii) below $20 \%$ oil phase, above $80 \%$ aqueous phase, and below $20 \%$ surfactant. There was an extensive region of TLS found at concentrations below $40 \%$ aqueous phase, above $45 \%$ oil phase, and the entire extent of the surfactant phase, and an extensive region of OS was found above $5 \%$ oil phase, below $30 \%$ surfactant, and below $70 \%$ aqueous phase. A similar diagram was constructed by Fonseca-Santos (19); however, he reported a larger region of phase separation. This may have occurred due the different method used by these authors to prepare the chitosan dispersion. In their study, chitosan was dispersed in a lower concentration of acetic acid than in our study, which may have led to a chitosan solution of lower solubility than expected, since it is well known that chitosan has low solubility at neutral or basic $\mathrm{pH}$, but can form soluble salts with organic acids such as acetic acid (19). In addition, the $\mathrm{pH}$ of chitosan is 6.5 , whereas the $\mathrm{pH}$ of oleic acid is 4.8 , and so therefore at the $\mathrm{pH}$ of the formulation $(\mathrm{pH}=5)$ used in our study, the chitosan will be positively charged and the oleic acid negatively charged; thus, it is likely that there will be an electrostatic interaction between the cationic groups of the amine portion of chitosan and the anionic groups of OA, leading to a greater stabilization of the formulation and, consequently, to a lower phase separation rate $(28,29)$.

Diagram 3 has not previously been reported and is illustrated in Fig. 1(3)) which depicts the systems obtained using PPG-5-CETETH-20 as the surfactant, OA as the oil 

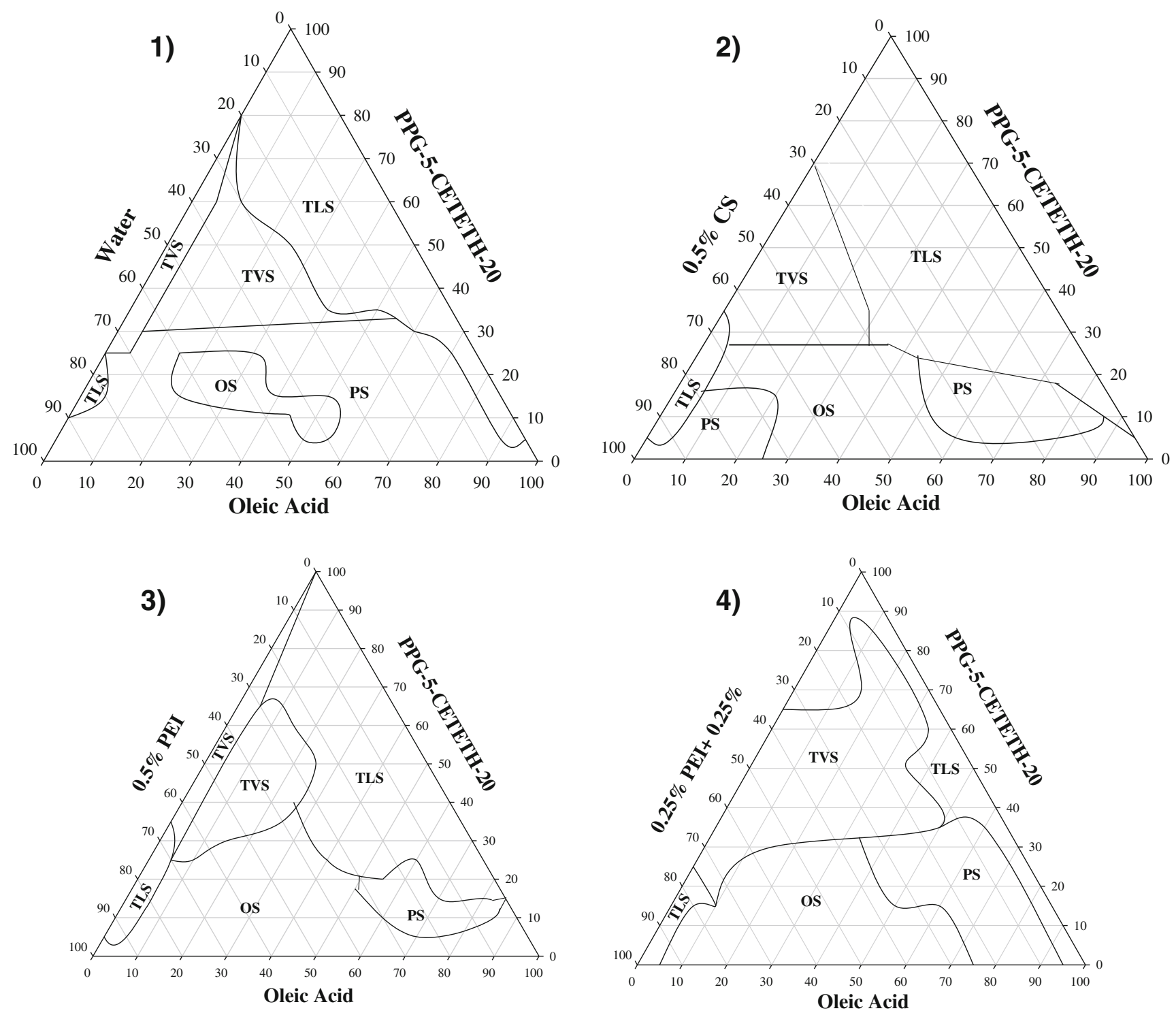

Fig. 1. Phase diagrams for PPG-5-CETETH-20, oleic acid, and (1)) water, (2)) chitosan (CS), (3)) polyethyleneimine (PEI)), and (4)) PEI + QS. TLS, transparent liquid system; TVS, transparent viscous system; OS, opaque system; and PS, phase separation

phase, and a dispersion of $0.5 \%$ PEI as the aqueous phase. There was a small phase separation region between 5 and $25 \%$ surfactants, above $50 \%$ oil phase, and below $30 \%$ aqueous phase. A TVS was obtained below $70 \%$ aqueous phase, below $20 \%$ oil phase, and above $25 \%$ surfactant. A TLS was formed in an extensive region above $15 \%$ surfactant, above $5 \%$ oil phase, and below $40 \%$ water. An OS was formed over the entire oil and aqueous phases, and below $30 \%$ surfactant. Overall, the diagram obtained was similar to diagram 2 , in that it showed less phase separation, which can be attributed to the large electrostatic interaction expected to occur between PEI and OA, since PEI has pKa between 9 and 10 and, therefore at an acid $\mathrm{pH}$, is positively charged, whereas the oleic acid is negatively charged (30).

Diagram 4 has also not been previously published and is illustrated in Fig. 1(4)) which represents the system obtained using PPG-5-CETETH-20 as the surfactant, OA as the oil phase, and a dispersion of $0.25 \%$ polyethyleneimine and
$0.25 \%$ chitosan dispersion as the aqueous phase. A phase separation region between 35 and $95 \%$ oil phases, below $35 \%$ aqueous phase, and below $40 \%$ surfactant was observed. There was an extensive TVS region between 10 and $75 \%$ aqueous phases, below $50 \%$ oil phase, and between 15 and $90 \%$ surfactants. There was also an OS region between 5 and $75 \%$ oil phases, above $25 \%$ aqueous phase, and below $40 \%$ surfactant. A narrow TLS region occurred above $85 \%$ oil phase, below 35\% aqueous phase, and throughout the total extent of the surfactant. This diagram contained the largest region for the TVS. Studies have shown that chitosan acts as a thickening agent by providing viscosity to formulations. However, when anions are added to the chitosan dispersion, its viscosity is decreased because of ion-dipole forces, i.e., the anions form a cascade of negative charge on the chitosan, generating repulsive forces between the chitosan molecules. This offers a low resistance to the flow and mobility of chitosan molecules, thereby decreasing viscosity. However, when the cationic PEI polymer is incorporated into the 
system, increases in viscosity may occur due to the increase in the total positive charge of the system (31).

Thus, with the addition of polymers to the aqueous phase of the system, a decrease in the extent of phase separation is observed, indicating that polymer addition led to a decrease in the coalescence of the system, as has previously been noted (32). It has also been reported that polymeric materials have the ability to modify the structural properties of drug delivery systems, due to the various cross-links present in their polymer chains that can result in mechanical and rheological changes in the system $(33,34)$.

When analyzing these four diagrams, it can be seen that from a surfactant concentration of $40 \%$, with the addition of water to the system, a transition from a liquid system to a viscous system tends to occur, and this is of special importance for LCPS for buccal administration $(8,35)$.

After performing this visual classification of the four different systems, all formulations classified as TLS and TVS were analyzed using polarized light microscopy (PLM).

PLM has become a widely used tool for the primary identification of micro-emulsions and mesophases in liquid crystalline systems $(36,37)$ since polarized light microscope has the ability to propagate the light beam in one single direction due to the presence of a polarizing light system coupled to its capacitor. Changes that a birefringent sample causes in the direction of propagation of the polarized light are detected by the analyzer, a second polarized light source, which is located next to the eyepiece. If the formulation is capable of altering the plane of polarized light, it is classified as anisotropic, and if the formulation fails to deflect the plane of polarized light, it is classified as isotropic (38).

The lamellar and hexagonal liquid crystalline mesophases are anisotropic, since when observed by polarized light microscopy, structures identified by the presence of Maltese crosses and striations were observed, respectively. In contrast, the cubic liquid crystalline mesophases and micro-emulsions (ME) were isotropic, and therefore, a dark field was obtained for these systems, but ME are TLSs, whereas the cubic crystalline liquid mesophases were TVSs (8). Thus, the classification of $\mathrm{ME}$ and cubic crystalline liquid mesophases is in conjunction with their viscosity $(39,40)$. After these PLM analyses, the four diagrams were reclassified delimiting these different mesophases, as shown in Fig. 2.

From these data, one formulation was selected from each diagram which had a low concentration of surfactant in order to decrease the toxic potential of the formulations (41). In addition, the formulation was selected as one being located in the transition area between the micro-emulsion and liquid crystalline system regions. This will allow the formulation to act as a liquid crystal precursor that, upon the addition of water (i.e., when it comes into contact with the saliva), can incorporate water from the saliva, forming a more organized liquid crystalline mesophase structure. This will allow for the formulation to provide more controlled drug release in the buccal cavity.

Thus, the formulations chosen were FW, FC, FP, and FPC, as shown in Fig. 2. These formulations were added to 30 and $100 \%$ saliva to investigate whether they could indeed exhibit LCPS behavior.
The nomenclature and composition of the selected formulations, with or without saliva, are described in Table I.

Figure 3 shows photomicrographs obtained from a PLM analysis of the formulations FW, FC, FP, and FPC in the presence and absence of saliva.

The formulations FW, FC, FP, and FPC were all isotropic, i.e., they were unable to deviate the plane of polarized light, suggesting the formation of a microemulsified system, since the apparent viscosity of the cubic liquid crystalline mesophase is extremely high $(39,40)$.

When $30 \%$ saliva was added to the FW, FC, FP, and FPC formulations, forming respectively the formulations FW30, FC30, FP30, and FPC30, Maltese cross-shaped formations were observed, suggesting that a transition to the more organized lamellar liquid crystalline mesophase had occurred.

When $100 \%$ saliva was added to the FW, FC, FP, and FPC formulations, forming respectively the formulations FW100, FC100, FP100, and FPC100, striated structures were observed suggesting that a transition to the more organized hexagonal liquid crystalline mesophase had occurred.

Carvalho et al. (27) have previously reported the development of a low-viscosity LCPS for the nasal administration of zidovudine (AZT). This formulation was composed of PPG-5CETETH-20, oleic acid, and water (55, 30, and 15\% w/w respectively). On contact with $100 \%$ artificial nasal mucus, the LCPS transformed into a lamellar liquid crystalline mesophase, that could act as a mucoadhesive matrix, thus promoting better nasal absorption of AZT, making it an interesting alternative for the treatment of acquired immunodeficiency. (27)

Salmazi et al. (8) have reported the development of a low-viscosity LCPS consisting of 40\% PPG-5-CETETH-20, $50 \%$ oleic acid, and $10 \%$ of a $0.5 \%$ chitosan dispersion containing $16 \%$ poloxamer, for vaginal administration of curcumin for the treatment of vaginal candidiasis. The addition of $100 \%$ artificial vaginal mucus to the lowviscosity LCPS resulted in the formation of a high-viscosity lamellar liquid crystalline system that had high mucoadhesion to the vaginal mucosa. In addition, the curcumin-loaded LCPS was shown to be 16 times more potent against Candida albicans when compared to free curcumin (8).

Dos Santos Ramos et al. (42) have developed an LCPS composed of $40 \%$ oleic acid, $40 \%$ PPG-5-CETETH-20, and $20 \%$ polymer dispersion for the incorporation of an extract of the medicinal plant Syngonanthus nitens Bong (Rhul.) This LCPS was examined pre-clinically for the treatment of vulvovaginal candidiasis using an in vivo study conducted in Wistar rats. The results were promising, as they demonstrated that this LCPS was transformed into a lamellar liquid crystalline mesophase upon the addition of $100 \%$ artificial vaginal mucus. The extract-loaded LCPS was found to be effective 2 days after the initiation of treatment and was more effective than the extract solution alone (42).

This mesophase transition occurs because of the polarization of the polar head of the PPG-5-CETETH-20 upon the addition of water which causes increases in the curvature and volume of the polar region, which then leads to the generation of hexagonal structures which are more effectively packed. Because these highly packed structures have more restricted lateral and translational movements, they have higher viscosity $(35,43)$. 

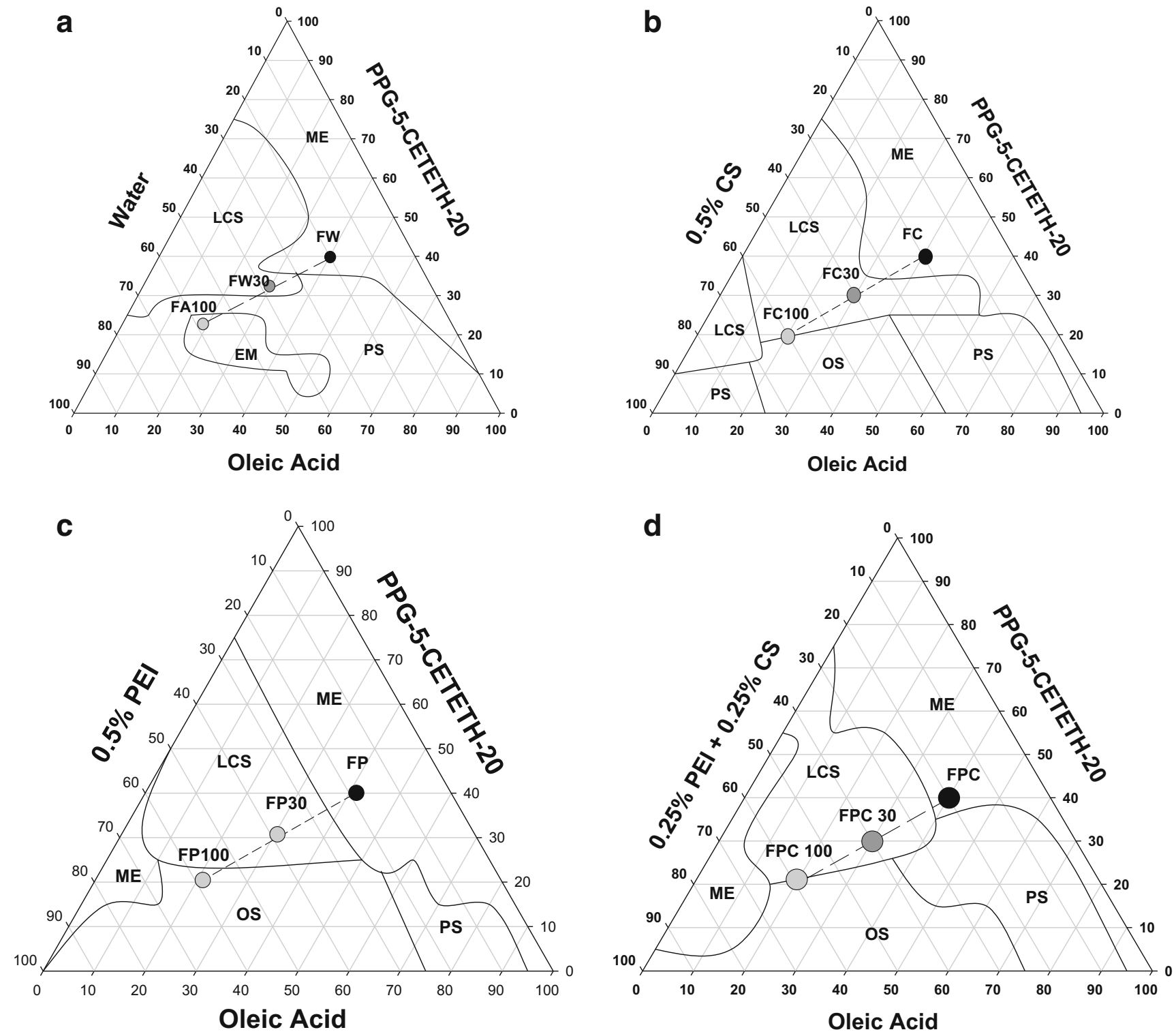

Fig. 2. Phase diagrams for PPG-5-CETETH-20, oleic acid (OA), and (A)) water, (B)) chitosan (CS), (C)) polyethyleneimine (PEI), and (D)) PEI + CS. LCS, liquid crystalline system; ME, micro-emulsion; PS, phase separation. FW, FC, FP, and FPC are the precursors of the liquid crystalline systems; FW30, FC30, FP30, and FPC30 are, respectively, the formulations FW, FC, FP, and FPC with 30\% artificial saliva. FW100, FC100, FP100, and FPC100 are, respectively, the formulations FW, FC, FP, and FPC with $100 \%$ artificial saliva

Table I. Nomenclature and composition (\%) of formulations with saliva (30 and 100\%) and without saliva

\begin{tabular}{|c|c|c|c|c|c|c|c|}
\hline Formulations & Oleic acid $(\%)$ & PPG-5-CETETH-20 (\%) & Water $(\%)$ & $5 \% \operatorname{CS}(\%)$ & $5 \%$ PEI $(\%)$ & $2.5 \% \mathrm{CS}+2.5 \%$ PEI $(\%)$ & Saliva $(\%)$ \\
\hline FW & 40 & 40 & 20 & - & - & - & - \\
\hline FW30 & 40 & 40 & 20 & - & - & - & 30 \\
\hline FW100 & 40 & 40 & 20 & - & - & - & 100 \\
\hline $\mathrm{FC}$ & 40 & 40 & 10 & 10 & - & - & - \\
\hline FC30 & 40 & 40 & 10 & 10 & - & - & 30 \\
\hline FC100 & 40 & 40 & 10 & 10 & - & - & 100 \\
\hline FP & 40 & 40 & 10 & - & 10 & - & - \\
\hline FP30 & 40 & 40 & 10 & - & 10 & - & 30 \\
\hline FP100 & 40 & 40 & 10 & - & 10 & - & 100 \\
\hline FPC & 40 & 40 & 10 & - & - & 10 & - \\
\hline FPC30 & 40 & 40 & 10 & - & - & 10 & 30 \\
\hline FPC100 & 40 & 40 & 10 & - & - & 10 & 100 \\
\hline
\end{tabular}




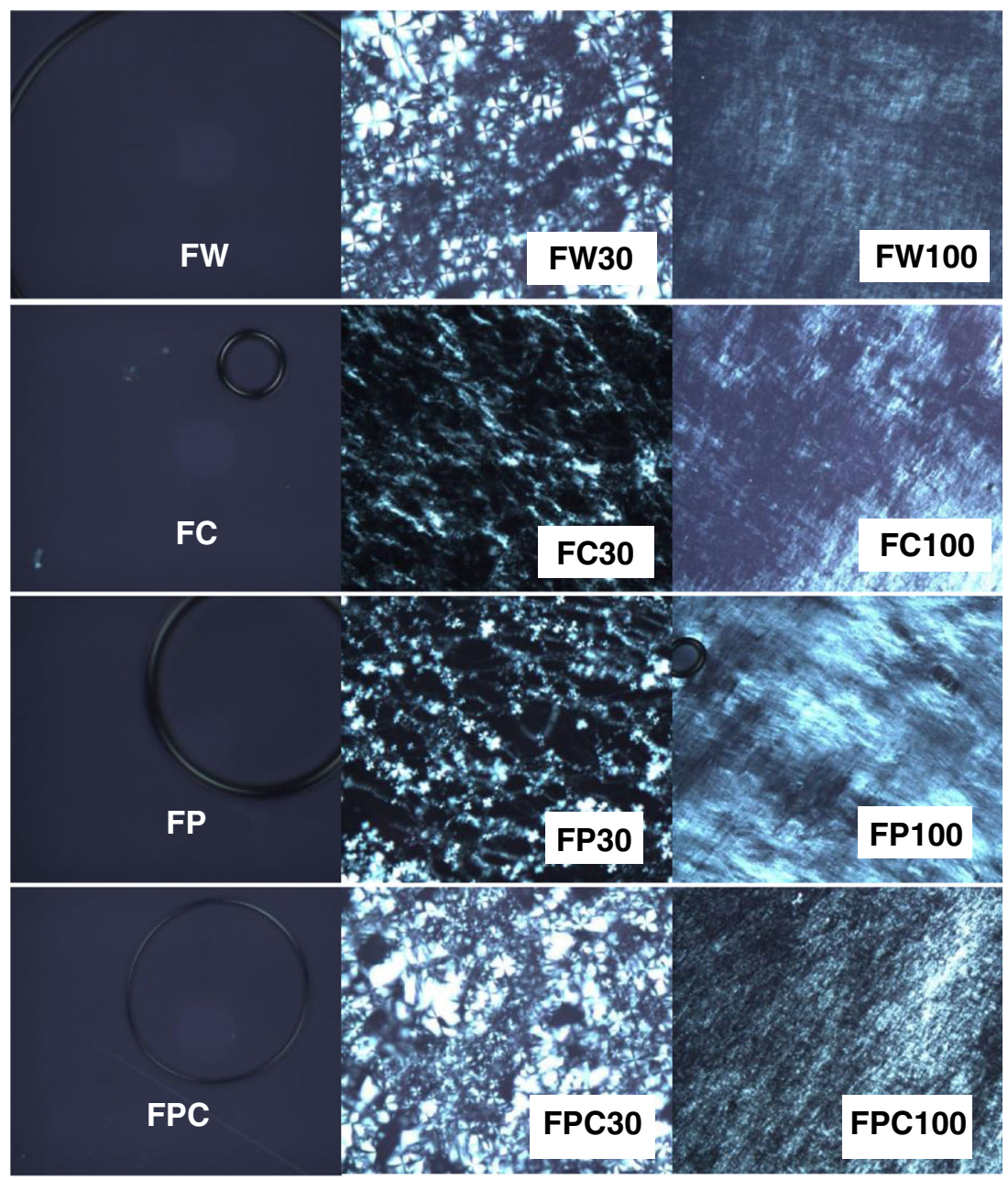

Fig. 3. Photomicrographs of the formulations FW, FW30, FW100, FC, FC30, FC100, FP, FP30, FP100, FPC, FPC30, and FPC100

These data therefore appear compatible with the proposed objective, since the chosen formulation from each diagram managed to behave as a precursor system for the generation of a liquid crystal phase upon the addition of saliva.

In order to confirm the structures obtained in the PLM, X-ray diffraction at angles of $\theta$ smaller than $10^{\circ}$, which correspond to the inter-planar distances on the nanoscale, has been shown to be effective in the characterization of systems formed by surfactant by the possibility of determining the mean size and distance between spreader objects, such as droplets, micelles, or liquid crystalline structures. Moreover, this technique allows one to evaluate the structure of scattering objects even if they are not organized in an orderly manner (44-46).

In micelle and micro-emulsion systems, SAXS curves have a broad band or peak, associated with a low threedimensional spatial correlation. Liquid crystalline systems with random orientations can aggregate, forming domains with one-, two-, and three-dimensional structures (47).

From the graphs of the SAXS analyses shown in Fig. 4, and the values obtained in Table II, it was possible to identify the mesophases of the liquid crystalline structures present.
A broad peak was observed in the SAXS curves for the formulations FW, FC, FP, FP30, and FPC, which shows the presence of micellar or micro-emulsion systems. On the other hand, the FW100 formulation had a ratio of 2:2.51, providing evidence for a hexagonal phase, which was supported by the structured striations seen in the photomicrographs from the polarized light dispersion analysis.

The formulations FW30, FPC30, and FC30 showed a correlation between the distances of the scattering objects with $d 1 / d 2=2$ and $d 1 / d 3=3$, showing a periodicity equivalent to a liquid crystalline arrangement of the lamellar phase, which was confirmed by the presence of Maltese crosses in the MLP.

The formulations FC100, FP100, and FPC100 also showed a 2:3 correlation which is characteristic of the lamellar mesophase. These samples are likely to be in a phase transition region, since striated structures were observed when observed by MLP, which confirms the idea that both the micro-emulsion system and liquid crystalline system are complex systems and hence, require more than one technique to characterize their structures $(48,49)$.

For micellar systems, the term $d$ is the separation found between rows of adjacent rod micelles. For the lamellar phases, $d$ is the spacing between two adjacent layers. For 

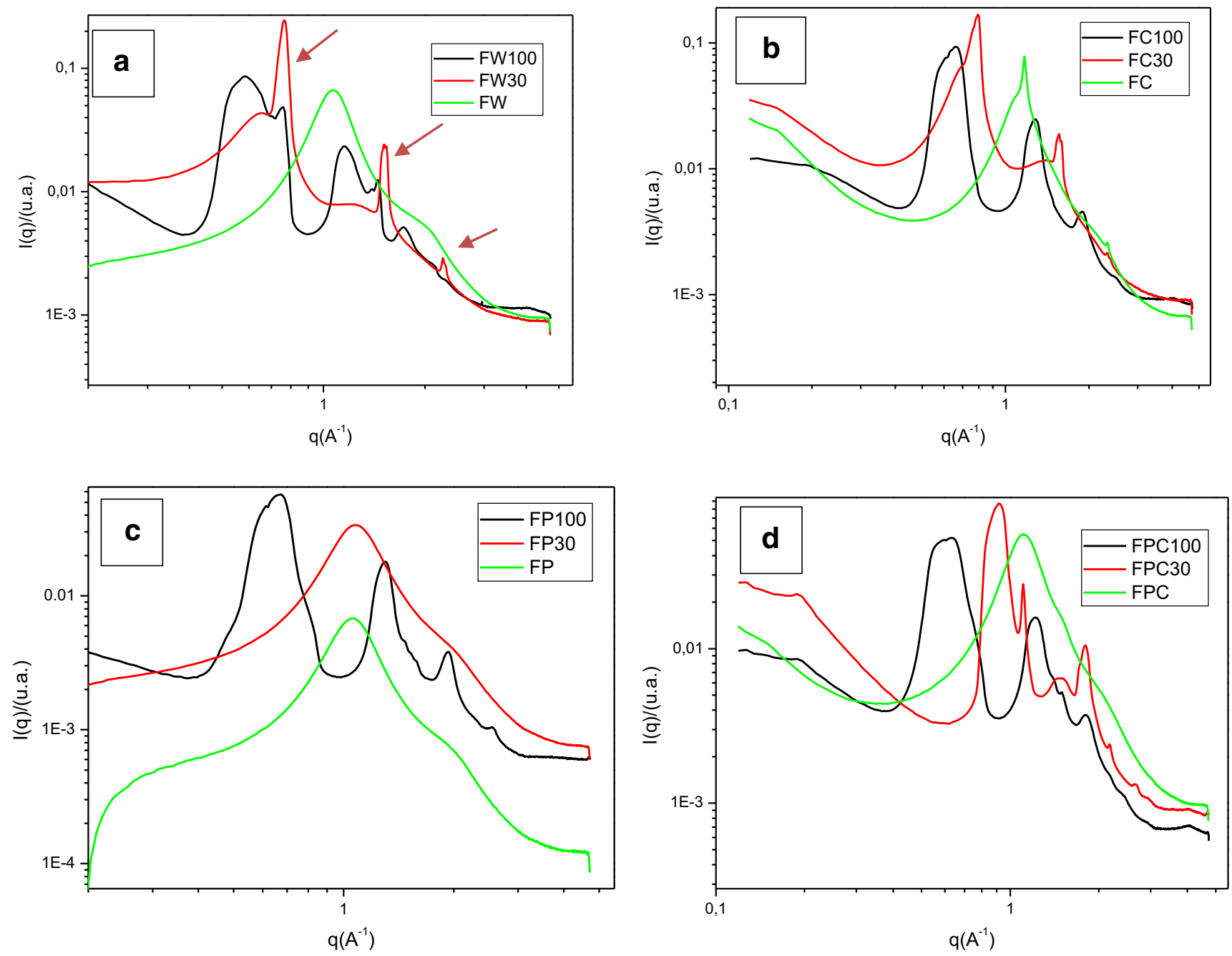

Fig. 4. Structural evaluation of formulations evaluated by SAXS: (A) FW, FW30, and FW100; (B) FC, FC30, and FC100; (C) FP, FP30, and FP100; (D) FPC, FPC30, and FPC100. The arrows in A indicate the Bragg peaks

hexagonal phases, $d$ is the distance between adjacent rows of cylinders in the hexagonal structure. The values of the distance between planes $(d)$ indicated in Table II are between 5 and $10 \mathrm{~nm}$, indicating that the structures of the formulations obtained are on the nanometer scale (50).
Following this, rheological studies were then conducted. The relationship between the shear stress and the shear rate for all the formulations is shown in Fig. 5.

The data values of the consistency index $(K)$ and flow behavior $(n)$, obtained by Eq. 1 , are shown in Table III.

Table II. Values of $q_{\max }(\AA)$ and ratio of distances $(d)$ between the inter-planar distances for the different formulations

\begin{tabular}{lllllll}
\hline Formulation & $q_{\max 1}$ & $q_{\max 2}$ & $q_{\max 3}$ & $d_{1} / d_{2}$ & $d_{1} / d_{3}$ & Structure \\
\hline FW & 1.06 & - & - & - & - & Micro-emulsion \\
FW30 & 0.76 & 1.52 & 2.28 & 2 & 3 & Lamellar \\
FW100 & 0.58 & 1.15 & 1.46 & 2 & 2.51 & Hexagonal \\
FC & 1.16 & - & - & - & - & Micro-emulsion \\
FC30 & 0.79 & 1.58 & 2.33 & 2 & 3 & Lamellar \\
FC100 & 0.66 & 1.27 & 1.90 & 2 & 3 & Lamellar \\
FP & 1.05 & - & - & - & - & Micro-emulsion \\
FP30 & 1.06 & - & - & - & - & Micro-emulsion \\
FP100 & 0.66 & 132 & 1.95 & 2 & -9.92 \\
FPC & 1.12 & - & - & - & - & Lamellar \\
FPC30 & 0.93 & 1.10 & 1.83 & 1 & 2 & Micro-emulsion \\
FPC100 & 0.61 & 1.21 & 1.83 & 2 & 3 & Lamellar \\
F & & & & & Lamellar
\end{tabular}



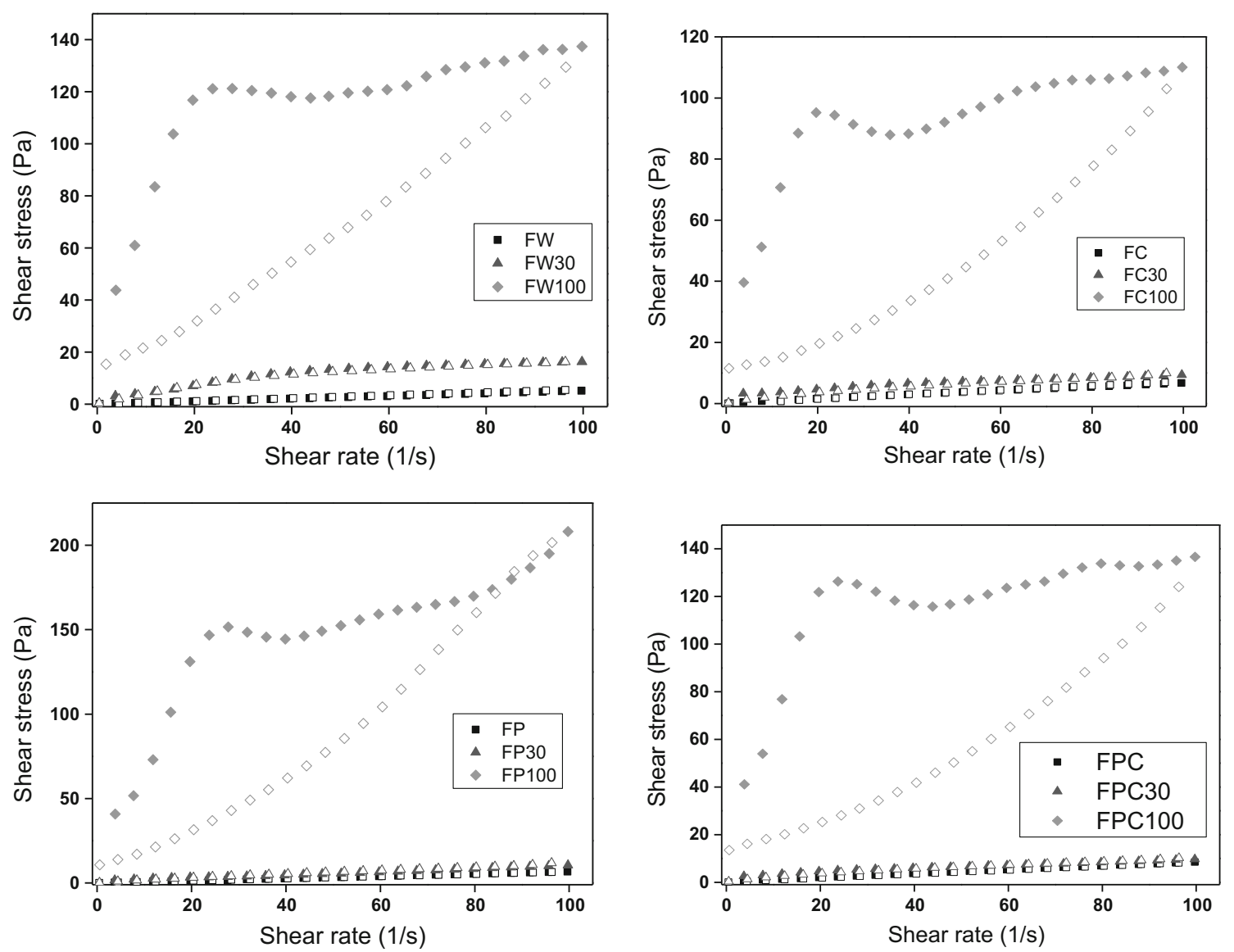

Fig. 5. Reograms of the different formulations. Full symbols curve upward and empty symbols curve downward

Statistical analysis by means and confidence intervals (CIs) was considered, and only the flow behavior indexes of the FW and FP formulations, in the absence of saliva, had $95 \%$ CIs that passed the standard value of 1 . All the other formulations showed values of $n$ less than 1 .

Therefore, these data indicate that only the FW and FP formulations had Newtonian flow behavior, whereas the other formulations showed non-Newtonian pseudoplastic behavior. In particular, the formulations FW100, FC100, FP100, and FPC100 present yield point, which is characteristic of plastic

Table III. Values of the consistency index $(K)$ and flow behavior $(n)$ of the formulations studied

\begin{tabular}{lll}
\hline Formulation & $K$ & $n$ \\
\hline FW & $0.052 \pm 0.010$ & $1.005 \pm 0.010$ \\
FW30 & $1.881 \pm 0.100$ & $0.468 \pm 0.024$ \\
FW100 & $48.194 \pm 2.961$ & $0.228 \pm 0.064$ \\
FC & $0.130 \pm 0.019$ & $0.905 \pm 0.023$ \\
FC30 & $1.565 \pm 0.452$ & $0.390 \pm 0.126$ \\
FC100 & $36.739 \pm 2.630$ & $0.241 \pm 0.027$ \\
FP & $0.063 \pm 0.018$ & $1.013 \pm 0.028$ \\
FP30 & $0.248 \pm 0.042$ & $0.862 \pm 0.051$ \\
FP100 & $36.244 \pm 1.759$ & $0.371 \pm 0.095$ \\
FPC & $0.128 \pm 0.016$ & $0.909 \pm 0.022$ \\
FPC30 & $1.418 \pm 0.160$ & $0.412 \pm 0.092$ \\
FPC100 & $60.424 \pm 3.418$ & $0.276 \pm 0.064$ \\
\hline
\end{tabular}

fluids. This behavior presents the same characteristics of the pseudoplastic flow, but with a yield stress, being necessary to overcome it so that the material begins to flow. Therefore, these materials require a greater external force that exceeds their internal cross-linking force to flow (51).

In addition, it was clear that the addition of saliva decreased the flow behavior index in a dose-dependent manner, increasing the pseudoplasticity of the formulations. This effect of the saliva appeared to be independent of the particular formulation.

Pseudoplastic behavior is a desirable property for mucoadhesive formulations that are intended for buccal drug delivery, since formulations with such behavior flow in the presence of an external force due to the alignment of their molecules towards the direction of fluid flow. During this process, solvent that had been previously entrapped in the molecular interlacings is released, and this decreases its resistance, which would be expected to enhance the spreadability of the formulation along the buccal mucosa. However, when this process ends, the formulation is expected to recover its initial form, i.e., a high-viscosity form, allowing it to be retained longer in the buccal mucosa, which would then increase drug bioavailability and subsequently, its local pharmacodynamic effect $(52,53)$.

In general, the addition of 30 or $100 \%$ saliva resulted in an increase in the mean consistency index, although the increase was more with the addition of $100 \%$ saliva.

Both formulation and saliva were found to have a highly significant effect on the consistency index, as well as the 
interaction between them. It was noticeable that the effect of saliva, formulation, and the interaction between them was high, suggesting that in practice, all have a high impact on the change in the consistency index.

The $K$ values were dependent on both the presence of saliva and the aqueous phase used. Thus, the gradual increase in saliva concentration gradually increased the consistency of all the formulations significantly. The addition of 30 or $100 \%$ saliva resulted in an increase in the mean value of the consistency index, although the increase was larger with the addition of $100 \%$ saliva. This is likely due to the fact that water present in the saliva causes solvation of the surfactant, increasing its packing factor, leading to the formation of more organized structures and consequently, increasing the consistency of the formulation (8).

On the other hand, the presence of CS and PEI in the aqueous phase resulted in the highest $K$ values, and the FPC formulation with $100 \%$ saliva (FPC100) had a significant 1.64 fold increase in the consistency index, relative to the FC100 formulation.

This increase in consistency index following the incorporation of polymers has already been reported in other continuous rheological studies in formulations intended for buccal administration. This can be explained by considering the numerous intermolecular bonds that occur between the polymer chains responsible for the viscosity and the thickening of the formulation $(52,54,55)$.
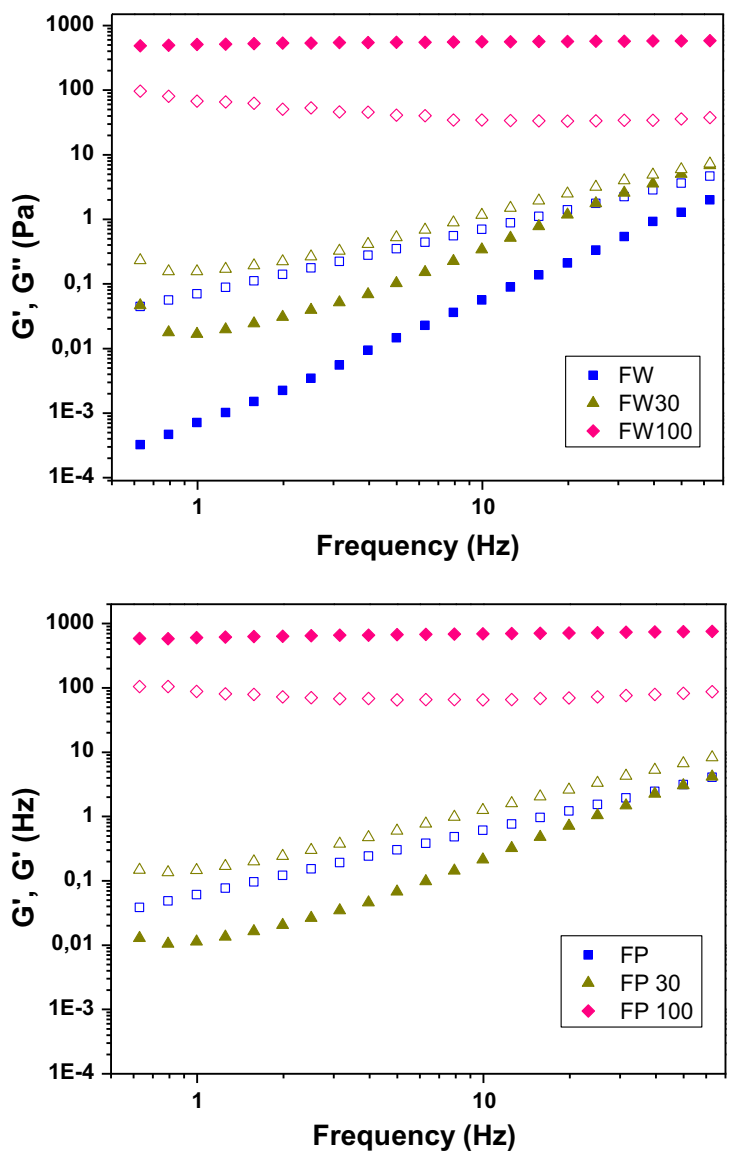

Thus, this increase in the consistency index shown by the FPC100 formulation may be due to an increased ionic interaction between the positive charges of the polymers and the negative charge of the saliva, resulting in a more cross-linked chitosan and polyethyleneimine gel, thus increasing the consistency of the formulations studied.

In an oscillatory rheological analysis, the shear stress varies as a sine wave, and the relationship between it and the resulting strain provides information on the viscoelasticity of the sample. Thus, an oscillatory rheological analysis was performed to examine the viscoelastic properties of the formulations, i.e., whether the formulation tends to be more viscous or more elastic. This data was used to provide information on the structural nature of the system, as it relates to the stability of the formulation. The oscillatory analyses of all the formulations are shown in Fig. 6.

It can be noted that, in general, the addition of 30 and $100 \%$ saliva in all formulations resulted in an increase in the mean values of the storage modulus $\mathrm{G}^{\prime}$, and in particular, a very significant increase was observed in the presence of $100 \%$ saliva for all the formulations.

Both the formulation and saliva had a highly significant effect on the storage modulus $\mathrm{G}^{\prime}$, as well as the interaction between them. It is of note that the effect of saliva, the formulation, and the interaction between them was high, suggesting that in practice, all of them have a high practical impact in $\mathrm{G}^{\prime}$, although the saliva effect appeared to be the most important. In addition, both the formulation and saliva
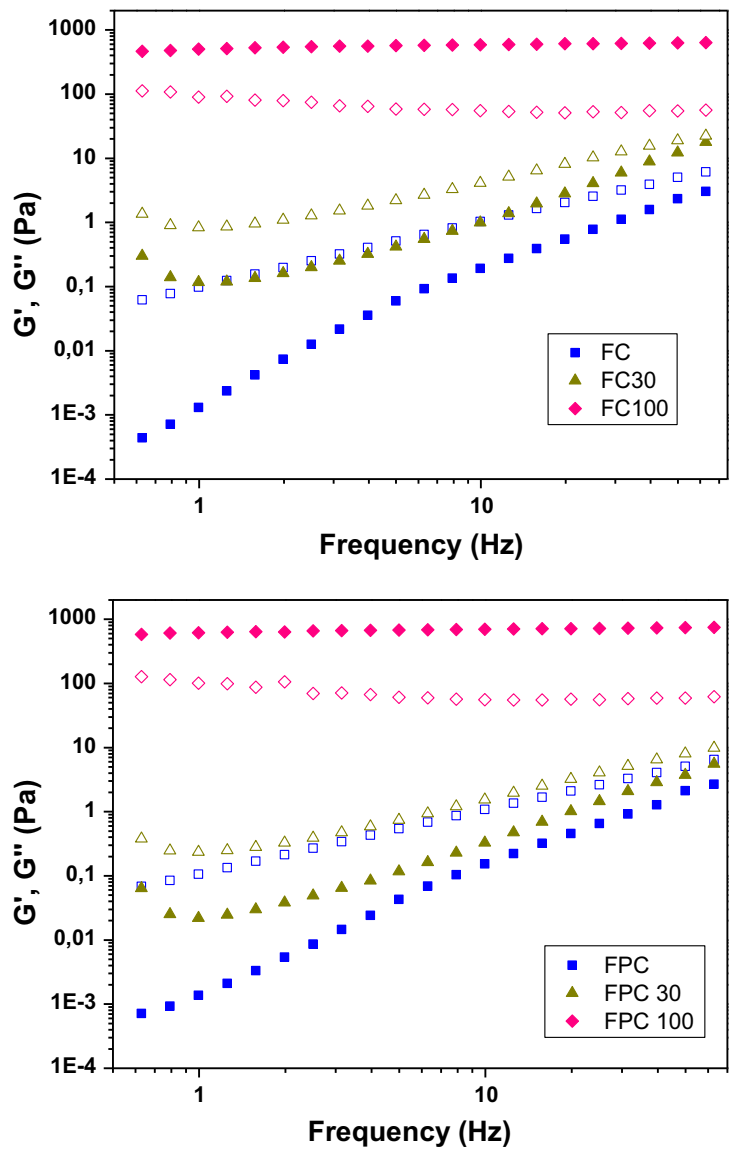

Fig. 6. Variation of storage modulus $\mathrm{G}^{\prime}$ (filled symbols) and loss $\mathrm{G}^{\prime \prime}$ (empty symbols) as a function of frequency for all formulations 
had a significant effect on the storage modulus $\mathrm{G}^{\prime}$, as well as the interaction between them.

For all the formulations, the increase in saliva promoted an increase in the mean values of $\mathrm{G}^{\prime \prime}$ and was considered statistically significant $(p \leq 0.001)$. In the absence of saliva, or in the presence of $30 \%$ saliva, the FPC formulation had values of $\mathrm{G}^{\prime \prime}$ significantly higher than the other formulations. In the presence of $100 \%$ saliva, the FPC formulation showed the highest value for the $\mathrm{G}^{\prime \prime}$ module, followed by FP.

In general, the addition of 30 or $100 \%$ saliva resulted in an increase in the mean values of the loss modulus $\mathrm{G}^{\prime \prime}$, and a very significant increase was observed in the presence of $100 \%$ saliva for all the formulations. In summary, formulations without saliva, as well as formulations with $30 \%$ saliva, were found to be more viscous than elastic $\left(G^{\prime \prime}>G^{\prime}\right)$. However, in the presence of $100 \%$ saliva, all the formulations were more elastic than viscous $\left(G^{\prime}>G^{\prime \prime}\right)$, having highly organized gel-like structures.

This behavior has also been reported in other oscillatory rheological studies performed on liquid crystal precursor systems, which demonstrated that after the addition of artificial mucus, viscous LCPSs could be transformed into liquid crystalline elastic systems. The hexagonal and cubic crystalline mesophases generally have a $\mathrm{G}^{\prime}$ greater than $\mathrm{G}^{\prime \prime}$, which reflects their high degree of structural organization $(8$, 42, 56).

Furthermore, all the formulations containing both chitosan and polyethyleneimine (FPC, FPC30, and FPC100) had the highest $G^{\prime}$ and $G^{\prime \prime}$ values, further indicating that the combination of these polymers in the aqueous phase was able to form a system with stronger crystalline structures. This behavior can be directly related to an increase in polymer entanglement, resulting in higher resistance of these formulations to deformation (57).

Considering the proposed buccal application for these formulations, it has been reported that elasticity is an important property to ensure resistance to deformation and therefore, good formulation stability, within the buccal mucosa. The formulations examined in this study appear to be appropriate for this purpose.

Analysis of the texture profile of the formulations reveals their mechanical properties, such as hardness, compressibility, tackiness, and cohesion.

The study of these parameters has become very useful in the development of pharmaceutical systems, since knowledge of these parameters can evaluate the effect of stresses found under physiological conditions, in addition to evaluating the structural characteristics of the formulations $(58,59)$, which can directly influence the clinical performance of the formulation.

Hardness is defined as the maximum resistance to compressive deformation, and compressibility is defined as the work required to compress the formulation. Hardness and compressibility show, respectively, the ease of application and the ease of spreading of the formulation on the biological surface to be treated $(45,58,59)$. In addition to these two parameters, we can also measure adhesiveness, which refers to the work required to overcome the connection between the formulation and the equipment probe. This parameter may involve breaking cohesive bonds within the formulation and so, may be related to sample cohesiveness. The greater the adhesion, the greater the cohesion of the formulation. Thus, these parameters are related to the adhesion of the formulation to the biological substrate and therefore, to the retention time of the formulation at the application site (60). A texture profile analysis was therefore performed on the formulations that showed mechanical resistance to flow, i.e., the formulations FW100, FP100, FPC100, and FC100. The data were analyzed statistically using a one-way ANOVA with a Tukey post-test at a significance level of $0.01 \%$ and are shown in Table IV.

There was no significant difference in hardness, compressibility, and adhesiveness between these formulations $(p>0.05)$. Jones et al. (58) also reported that they did not observe an increase in mechanical parameters when the polymer concentration in cellulose gels increased because of the high cohesive binding strength in these samples which prevented the formation of adhesive interactions with the probe (58).

Importantly, however, all the formulations were statistically different from the commercial formulation. All of the formulations had twice the hardness and compressibility values of the commercial formulation, which is interesting because compressibility is a measure of the work required to break the physical bonds within the formulation. These data therefore indicate that molecules within the formulation have rigid bonds with each other which increased the physical stability of the formulation (61).

Adhesiveness, which is the ability to remove the probe from the formulation, was also twice as high for the developed formulations, compared to the commercial formulation, indicating that they have a higher adhesive power, which is considered to be a very important property in developing a buccal formulation. Bruschi et al. (6) have developed semi-solid formulations for periodontal applications with increased adhesiveness and reported that this feature helps to provide better retention of the formulation within the periodontal pocket (6).

Table IV. Mechanical properties of formulations determined by texture profile analysis. Each value represents the mean \pm standard deviation at a temperature of $25^{\circ} \mathrm{C}$

\begin{tabular}{lllll}
\hline Formulation & Hardness $(\mathrm{mN})$ & Compressibility $(\mathrm{mN} \mathrm{s})$ & Adhesiveness $(\mathrm{mN} \mathrm{s})$ & Cohesion (dimensionless) \\
\hline FW 100 & $14.70 \pm 1.15$ & $108.55 \pm 5.57$ & $82.1 \pm 3.80$ & $0.72 \pm 0.0049$ \\
FP 100 & $15.33 \pm 0.19$ & $111.00 \pm 4.03$ & $78.6 \pm 4.24$ & $0.71 \pm 0.0028$ \\
FC 100 & $12.69 \pm 2.90$ & $99.07 \pm 16.10$ & $69.5 \pm 2.12$ & $0.70 \pm 0.0082$ \\
FPC100 & $14.26 \pm 2.42$ & $105.81 \pm 16.24$ & $67.2 \pm 1.90$ & $0.69 \pm 0.0022$ \\
Commercial & $7.08 \pm 1.80$ & $49.60 \pm 4.10$ & $35.2 \pm 2.65$ & $0.79 \pm 0.0020$ \\
\hline
\end{tabular}




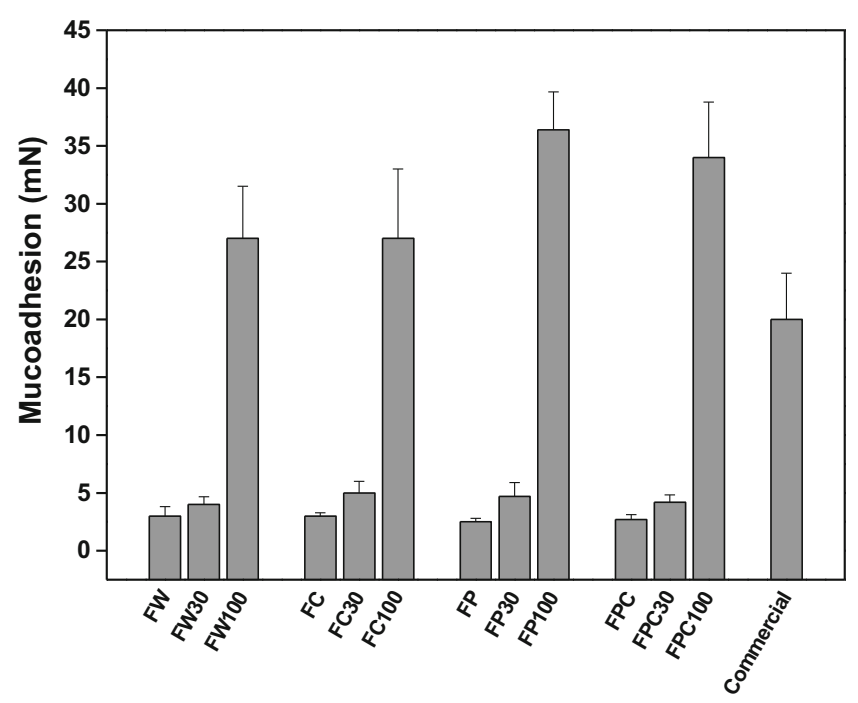

Formulation

Fig. 7. Mucoadhesion $(\mathrm{mN})$ of the different formulations measured at $37^{\circ} \mathrm{C}$. Each value represents the mean \pm standard deviation

Buccal drug administration is a non-invasive route of drug administration, which has gained much interest in recent years. The buccal membrane is multilayered and is a nonkeratinized mucosa rich in underlying blood vessels that has good drug permeability through transcellular and paracellular pathways. However, the main challenge related to buccal administration of drugs is the length of time the formulation remains in the buccal mucosa, since saliva production, food intake, mouth movement, and swallowing may prevent the formulation from adhering to the buccal mucosa. These factors could lead to a reduction in, or the absence of, therapeutic efficacy. Thus, the ideal buccal drug delivery system should exhibit mucoadhesion to the buccal mucosa coupled with sufficient stability to be efficacious.

Mucoadhesion is defined as the state in which two materials, at least one of a biological nature, are held together for an extended period of time by interfacial tension (62). The main advantage of using adhesive bio (muco) systems as drug carriers is to prolong the residence time of the drug at the site of application, which allows for enhanced contact of the formulation with the biological barrier, thereby decreasing the frequency of application of the product and increasing patient compliance (10). The mucoadhesion strength for all the formulations is shown in Fig. 7.

The incorporation of $30 \%$ saliva into the formulations significantly increased their mucoadhesion, and this was true for all of the different formulations. The incorporation of $100 \%$ saliva significantly increased mucoadhesion compared to formulations without saliva, or those containing 30\% saliva, irrespective of the formulation.

The formulations FC100, FP100, and FPC100 achieved equal levels of mucoadhesion; however, the FP100 formulation was the only one that had a significant difference in mucoadhesion difference, compared to the formulation using the aqueous phase (FA100).

We anticipated that the FPC100 formulation would have the highest mucoadhesion value, since formulations with high consistency index and storage modulus $\left(\mathrm{G}^{\prime}\right)$ values tend to have high mucoadhesion values. However, other studies have suggested that formulations with a high degree of internal stiffness may impair the interaction with proteins in the biological membrane, since the interaction between polymers and mucosal proteins is one of the key factors of mucoadhesion $(8,44)$. Importantly, the FPC formulation was approximately twice as mucoadhesive as the commercial formulation used for the buccal administration of drugs.

A drug delivery system may be defined as a formulation that allows the introduction of a therapeutic substance into the organism, improving its effectiveness and safety by controlling the rate, time, and target in the organism. Therefore, to evaluate the drug release profile of the predeveloped formulation is a critical factor to select a formulation that enhances the drug bioavailability. Finally, this study can also predict the in vivo behavior of the drug delivery system $(63,64)$.

Thus, it was incorporate the peptide CTT1 into FW (FWCTT1) and FPQ (FPQ-CTT1) to investigate how these both LCPSs can control the drug release.

Peptidic drugs exhibit physico-chemical characteristics that may affect their biological action, such as poor intestinal permeability due to their high molecular mass as well as low oral bioavailability (65), because of the first-pass hepatic metabolism. Thus, it is necessary the use of the parenteral route, which may present a series of drawbacks, such as the need for repeated injections due to its reduced half-life (66), and the occurrence of side effects such as thrombophlebitis and tissue necrosis (67). Therefore, the incorporation of CTT1 into a clinically adequate, safe, and effective delivery system aiming at its buccal administration proves to be an interesting option to make its use feasible in the treatment of diseases.

The release profile of the CTT1 peptide is shown in Fig. 8.

The peptide in solution was $65 \%$ released within $24 \mathrm{~h}$; however, only $7 \%$ of the peptide was released from the FW formulation and $3 \%$ of the peptide was released from the FPC formulation in $24 \mathrm{~h}$.

These results show that both formulations behaved as a controlled drug delivery system, with the polymer

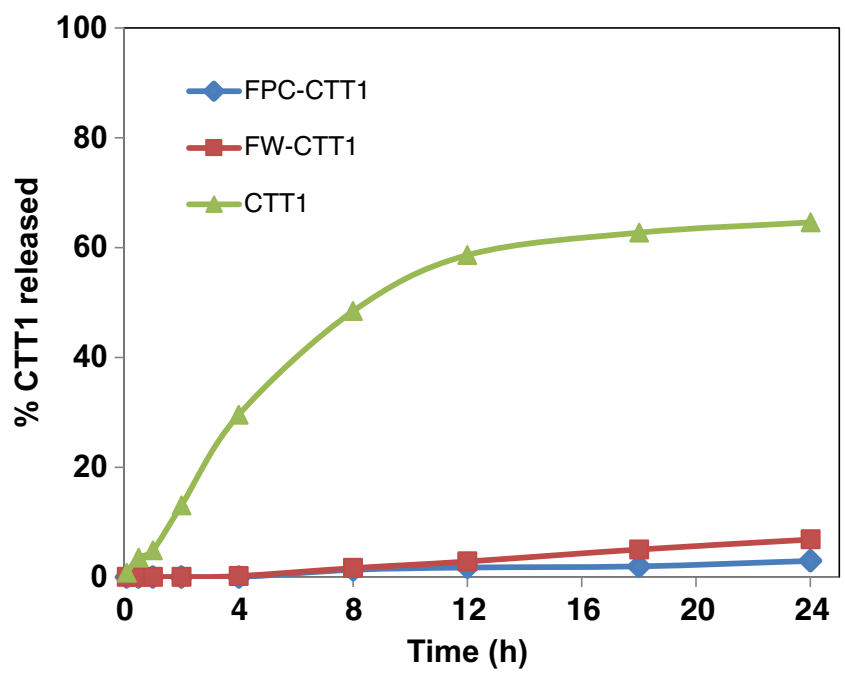

Fig. 8. The in vitro release profiles of CTT1in solution (CTT1), CTT1-loaded FPC (FPC-CTT1), and CTT1-loaded FW (FW-CTT1). The values represent the mean $\pm \mathrm{SD}$ of six replicates 
Table V. The adjusted parameters of the kinetic models used in the release of the CTT1 from the formulations

\begin{tabular}{|c|c|c|}
\hline \multirow[t]{2}{*}{ Mathematical models } & \multicolumn{2}{|c|}{ Formulations } \\
\hline & FW-CTT1 & FPC-CTT1 \\
\hline \multicolumn{3}{|l|}{ Korsmeyer-Peppas } \\
\hline$R^{2}$ & 0.9964 & 0.9718 \\
\hline$n$ & 1.3599 & 1.0388 \\
\hline \multicolumn{3}{|l|}{ Higuchi } \\
\hline$R^{2}$ & 0.8662 & 0.8912 \\
\hline$K$ & 0.9994 & 0.4660 \\
\hline \multicolumn{3}{|l|}{ First order } \\
\hline$R^{2}$ & 0.9814 & 0.9716 \\
\hline$K$ & 0.0027 & 0.0012 \\
\hline \multicolumn{3}{|l|}{ Weibull } \\
\hline$R^{2}$ & 0.9998 & 0.9907 \\
\hline$b$ & 0.6780 & 0.6328 \\
\hline
\end{tabular}

formulation further controlling the release of the drug. This can be attributed to the formation of a more compact and high-density cross-linking structure, reducing the release capacity of the drug to the dissolution medium, promoting greater release control. This corroborates the rheological data that demonstrated that the FPC formulation is highly elastic. de Oliveira Cardoso et al. (66) also demonstrated that more elastic formulations further control drug release (68).

The phenomena involved in the release of drugs from drug delivery systems are very complex, so the mathematical study of release kinetics may provide data for a better understanding of the drug releasing profiles. Therefore, it was investigated which release model (Korsmeyer-Peppas, Higuchi, Weibull, or first order) is more suitable for formulations. The adjusted data for the selected kinetic models are listed in Table V.

Based on the higher values of adjusted $R^{2}$, it was found that for all the formulations studied, Weibull was the model that best correlated with drug release.

Weibull model is considered the most versatile model because it allows a better adjustment to the different types of release profiles. This model is based on Eq. 3 below:

$\frac{M_{t}}{M_{\infty}}=1-\exp \left(-a t^{b}\right)$

where $M_{t}$ is the cumulative amount of drug released at time $t$ and $M_{\infty}$ is the cumulative amount of drug released at infinity; $a$ is the scale parameter and $b$ is the shape parameter (69).

The value of $b$ is related to the transport mechanism of the drug through the polymer matrix. In this way, $b$ is the parameter of form that characterizes the curve as being exponential $(b=1$, case $1)$, sigmoid ( $b>1$, case 2$)$, or parabolic $(b<1$, case 3$)$. The value of $b$ for all the studied samples was less than 0.75 , indicating that all the samples release the drug through Fickian diffusion, which postulates that drug fluxes go from high-concentration regions to low-concentration regions, with a magnitude proportional to the concentration gradient (70). These values are consistent with case 3 which exhibits a higher initial inclination followed by an exponential curvature, as is evident from the release profiles for the formulations of Fig. 8.

Carvalho et al. (11) observed a similar behavior when investigating the release of AZT incorporated into liquid crystal precursor systems for nasal mucosa. They reported that the release mechanism of all formulations followed the Weibull model; however, the value of $b$ was dependent on the degree of organization of the systems, that is, the formulations with low consistency index, which presented similar values to the formulations developed in that study, presented a value of $b<0.75$, while the most organized formulations had a value of $b>1.0$. This is because in Fickian diffusion, an increase in the value of $b$ reflects in disorder of the formulation. These data are in agreement with our results that demonstrated that the FPC formulation had a consistency value greater than FW; however, these values are low and therefore, the formulations follow the same release mechanism (11).

Based on statistical analyses of the physical tests performed by us, we concluded that the FPC formulation behaves as the best LCPS with potential interesting for buccal administration of drugs. As a result, FPC was selected to perform a cytotoxicity test, and the data are shown in Fig. 9.

The results demonstrate that at concentrations above $0.06 \mathrm{mg} / \mathrm{mL}$, the FPC formulation significantly decreased the cell viability of strains, indicating a cytotoxic effect of the FPC formulation. Studies have shown that most drugs or devices designed for buccal mucosal application have a cytotoxic potential. However, the clinical relevance of a cell culture toxicity result is often difficult to interpret, since the buccal mucosa is generally more resistant to toxic substances than a cell culture, because of the presence of mucin and keratin layers, and so we do not think that result necessarily reflects the risk of long-term adverse effects with FPC (71).

Thus, it was possible to develop a novel liquid crystalline system composed of PPG-5-CETETH-20, oleic acid, chitosan, and PEI with safe and satisfactory mucoadhesive, rheological, and mechanical characteristics for buccal administration of drugs.

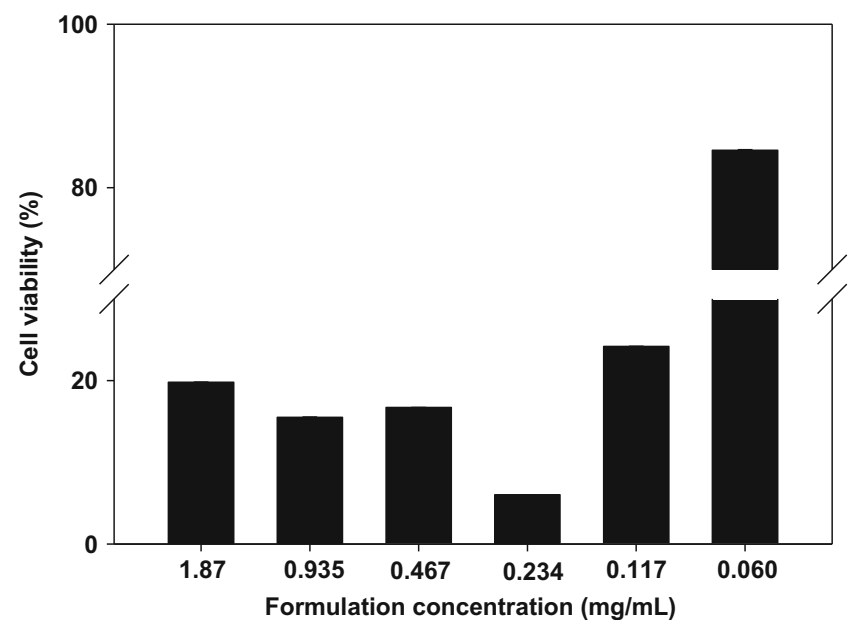

Fig. 9. Cellular viability (\%) of FPC for HACAT strains after $48 \mathrm{~h}$ of treatment 


\section{CONCLUSIONS}

The data show that it is possible to develop a precursor of liquid crystalline system composed of chitosan and polyethyleneimine as the aqueous phase, oleic acid as the oil phase, and ethoxylated and propoxylated cetyl alcohol as the surfactant for use as drug delivery system for the buccal route. Both MLP and SAXS analyses provided evidence for an increase in viscosity and the adoption of a liquid crystalline state following incorporation of saliva. Rheological, mechanical, and mucoadhesive tests demonstrated that the incorporation of both saliva and polymers created a more structured and adhesive LCS in situ. In vitro drug release study showed that the FPC was able to control the release of the model drug, which can improve the therapy outcome. In vitro cytotoxicity tests demonstrated that a low concentration of the FPC formulation did not exhibit cytotoxicity; therefore, the data presented here provide a novel LCS for buccal drug delivery for biomedical applications in the treatment of several buccal or systemic diseases.

\section{ACKNOWLEDGEMENTS}

We thank the Grant No. 2013/01565-1 from the Fundação de Amparo à Pesquisa do Estado de São Paulo (FAPESP), Conselho Nacional de Desenvolvimento Científico e Tecnológico (CNPq), and Programa de Apoio ao Desenvolvimento Científico (PADC).

\section{COMPLIANCE WITH ETHICAL STANDARDS}

Conflict of Interest The authors declare that they no conflicts of interest.

\section{REFERENCES}

1. Şenel S, Kremer MJ, Kaş S, Wertz PW, Hıncal AA, Squier CA. Enhancing effect of chitosan on peptide drug delivery across buccal mucosa. Biomaterials. 2000;21:2067-71.

2. Hearnden V, Sankar V, Hull K, Juras DV, Greenberg M, Kerr $\mathrm{AR}$, et al. New developments and opportunities in oral mucosal drug delivery for local and systemic disease. Adv Drug Deliv Rev. 2012;6:16-28.

3. Kang ML, Cho CS, Yoo HS. Application of chitosan microspheres for nasal delivery of vaccines. Biotechnol Adv. 2009;27:857-65.

4. Formariz TP, Chiavacci LA, Sarmento VHV, Franzini CM, Silva-Jr AA, Scarpa MV, et al. Structural changes of biocompatible neutral microemulsions stabilized by mixed surfactant containing soya phosphatidylcholine and their relationship with doxorubicin release. Colloids Surf B Biointerfaces. 2008;63:287-95.

5. Urban MCC. Desenvolvimento de sistemas de liberação micro e nanoestruturados para administração cutânea do acetato de dexametasona [dissertation]. Araraquara (SP): Sao Paulo State University, 2004.

6. Bruschi ML, Jones DS, Panzeri H, Gremião MP, De Freitas O, Lara EH. Semisolid systems containing propolis for the treatment of periodontal disease: in vitro release kinetics, syringeability, rheological, textural, and mucoadhesive properties. J Pharm Sci. 2007;96:2074-89.
7. Chorilli M, Prestes PS, Rigon RB, Leonardi GR, Chiavacci LA, Scarpa MV. Development of liquid-crystalline systems using silicon glycol copolymer and polyether functional siloxane. Quim Nova. 2009;32:1036-40.

8. Salmazi R, Calixto G, Bernegossi J, dos Santos Ramos MA, Bauab TM, Chorilli M. A curcumin-loaded liquid crystal precursor mucoadhesive system for the treatment of vaginal candidiasis. Int J Nanomedicine. 2015;10:4815.

9. Calixto GMF, Garcia MH, Cilli EM, Chiavacci LA, Chorilli M. Design and characterization of a novel p1025 peptide-loaded liquid crystalline system for the treatment of dental caries. Molecules. 2016;21:158.

10. Croda do Brasil. Folha de informações de segurança de produto químico - FISPQ. Procetyl AWS: revisão no1 de 29/01/2002 de acordo com NBR 14725. Campinas, 2002. 3p.

11. Carvalho FC, Sarmento VH, Chiavacci LA, Barbi MS, Gremião MP. Development and in vitro evaluation of surfactant systems for controlled release of zidovudine. J Pharm Sci. 2010;99:2367-74.

12. Smart JD, Riley RG, Tsibouklis J, Young SA, Hampson FC, Davis JA, et al. The retention of 14 C-labelled poly (acrylic acids) on gastric and oesophageal mucosa: an in vitro study. Eur J Pharm Sci. 2003;20:83-90.

13. Jiang HL, Kim YK, Arote R, Nah JW, Cho MH, Choi YJ, et al. Chitosan-graft-polyethylenimine as a gene carrier. J Control Release. 2007;117:273-80.

14. Jere D, Jiang HL, Kim YK, Arote R, Choi YJ, Yun CH, et al. Chitosan-graft-polyethylenimine for Akt1 siRNA delivery to lung cancer cells. Int J Pharm. 2009;378:194-200.

15. van der Lubben IM, Verhoef JC, Borchard G, Junginger HE. Chitosan and its derivatives in mucosal drug and vaccine delivery. Eur J Pharm Sci. 2001;14:201-7.

16. Günther M, Lipka J, Malek A, Gutsch D, Kreyling W, Aigner A. Polyethylenimines for RNAi-mediated gene targeting in vivo and siRNA delivery to the lung. Eur J Pharm Biopharm. 2011;77:438-49.

17. Fonseca-Santos B, Satake CY, Calixto GMF, Santos AM, Chorilli M. Trans-resveratrol-loaded nonionic lamellar liquidcrystalline systems: structural, rheological, mechanical, textural, and bioadhesive characterization and evaluation of in vivo antiinflammatory activity. Int J Nanomedicine. 2017;12:6883-93.

18. Bernegossi J. Desenvolvimento, caracterização e ação antibiofilme oralde sistemas nanoestruturados bioadesivos contendo o peptídeo KSL-W. Araraquara (SP): Sao Paulo State University; 2014.

19. Fonseca-Santos B. Sistemas precursores de cristais líquidos mucoadesivos para administração bucal de curcumina no tratamento do câncer bucal. Araraquara (SP): Sao Paulo State University; 2015.

20. Bernegossi J, Calixto GMF, Sanches PRDS, Fontana CR, Cilli EM, Garrido SS, et al. Peptide KSL-W-loaded mucoadhesive liquid crystalline vehicle as an alternative treatment for multispecies oral biofilm. Molecules. 2015;21:37.

21. Cintra GADS, Pinto LA, Calixto GMF, Soares CP, Von Zuben EDS, Scarpa MV, et al. Bioadhesive surfactant systems for methotrexate skin delivery. Molecules. 2016;21:231.

22. Turunen TM, Urtti A, Paronen P, Audus KL, Rytting JH. Effect of some penetration enhancers on epithelial membrane lipid domains: evidence from fluorescence spectroscopy studies. Pharm Res. 1994;11:288-94.

23. Ganem-Quintanar A, Quintanar-Guerrero D, Falson-Rieg F, Buri P. Ex vivo oral mucosal permeation of lidocaine hydrochloride with sucrose fatty acid esters as absorption enhancers. Int J Pharm. 1998;173:203-10.

24. Marques MR, Loebenberg R, Almukainzi M. Simulated biological fluids with possible application in dissolution testing. Dissolut Technol. 2011;18:15-28.

25. Lawrence MJ, Rees GD. Microemulsion-based media as novel drug delivery systems. Adv Drug Deliv Rev. 2000;45:89-121.

26. Feigenson GW, Buboltz JT. Ternary phase diagram of dipalmitoyl-PC/dilauroyl-PC/cholesterol: nanoscopic domain formation driven by cholesterol. Biophys J. 2011;80:2775-88.

27. Carvalho FC, Campos ML, Peccinini RG, Gremião MPD. Nasal administration of liquid crystal precursor mucoadhesive vehicle as an alternative antiretroviral therapy. Eur J Pharm Biopharm. 2013;84:219-27. 
28. Casettari L, Illum L. Chitosan in nasal delivery systems for therapeutic drugs. J Control Release. 2014;190:189-200.

29. Ham-Pichavant F, Sèbe G, Pardon P, Coma V. Fat resistance properties of chitosan-based paper packaging for food applications. Carbohydr Polym. 2005;61:259-65.

30. Ricardo NMPS, Ricardo NMPS, Costa Fde MLL, Bezerra FWA, Chaibundit C, Hermida-Merino D, et al. Effect of water-soluble polymers, polyethylene glycol and poly (vinylpyrrolidone), on the gelation of aqueous micellar solutions of Pluronic copolymer F127. J Colloid Interface Sci. 2012;368:336-41.

31. Abid CKV, Jain S, Jackeray R, Chattopadhyay S, Singh H. Formulation and characterization of antimicrobial quaternary ammonium dendrimer in poly (methyl methcarylate) bone cement. J Biomed Mater Res B Appl Biomater. 2017;105:52130 .

32. Klinkesorn U, Sophanodora P, Chinachoti P, McClements DJ. Stability and rheology of corn oil-in-water emulsions containing maltodextrin. Food Res Int. 2004;37:851-9.

33. Ferreira SBDS, Moço TD, Borghi-Pangoni FB, Junqueira MV, Bruschi ML. Rheological, mucoadhesive and textural properties of thermoresponsive polymer blends for biomedical applications. J Mech Behav Biomed Mater. 2016;55:164-78.

34. Carvalho FC, Chorilli M, Gremião MPD. Nanotechnologybased polymeric bio (muco) adhesive platforms for controlling drug delivery-properties, methodologies and applications. Polímeros. 2014;24:203-13.

35. Malmsten M. Surfactants and polymers in drug delivery. New York: Infoma Healthcare; 2002.

36. Oyafuso MH, Carvalho FC, Takeshita TM, de Souza ALR, Araújo DR, Merino V, et al. Development and in vitro evaluation of Lyotropic liquid crystals for the controlled release of Dexamethasone. Polymers. 2017;9:330.

37. Chorilli M, Prestes PS, Rigon RB, Leonardi GR, Chiavacci LA, Sarmento VHV, et al. Structural characterization and in vivo evaluation of retinyl palmitate in non-ionic lamellar liquid crystalline system. Colloids Surf, B. 2011;85:182-8.

38. Shah HJ, Fontecchio AK, Mattia D, Gogotsi Y. Field controlled nematic-to-isotropic phase transition in liquid crystal-carbon nanotube composites. J Appl Phys. 2008;103:064314.

39. Gabboun NH, Najib NM, Ibrahim HG, Assaf S. Release of salicylic acid, diclofenac acid and diclofenac acid salts from isotropic and anisotropic nonionic surfactant systems across rat skin. Int J Pharm. 2001;212:73-80.

40. Hyde ST. Identification of lyotropic liquid crystalline mesophases. In: Krister Holmberg, editor. Handbook of applied surface and colloid chemistry. Wiley; 2001. p. 299-332.

41. Klein T. Desenvolvimento de sistemas nanoestruturados estabilizados com álcool cetilico etoxilado e propoxilado contendo fluconazol potencialmente ativo contra esporotricose. [dissertation]. Araraquara (SP): Sao Paulo State University; 2007.

42. dos Santos Ramos MA, de Toledo LG, Calixto GMF, Bonifácio BV, de Freitas Araújo MG, dos Santos LC, et al. Syngonanthus Nitens bong.(Rhul.)-loaded Nanostructured system for Vulvovaginal Candidiasis treatment. Int J Mol Sci. 2016;17:1368.

43. Mezzenga R. Physics of self-assembly of Lyotropic liquid crystals. In: Garti N, Somasundaran P, Mezzenga R, editors. Self-assembled supramolecular architectures: lyotropic liquid crystals. Hoboken: Wiley; 2012. p. 1-20.

44. Manaia EB, Kaminski RCK, Oliveira AG, Corrêa MA, Chiavacci LA. Multifunction hexagonal liquid-crystal containing modified surface $\mathrm{TiO} 2$ nanoparticles and terpinen-4-ol for controlled release. Int J Nanomedicine. 2015;10:811-9.

45. Carvalho FC, da Luz GM, da Silva Barbi M, Landgraf DS, Chiavacci LA, Sarmento VHV, et al. Rheological, mechanical and adhesive properties of surfactant-containing systems designed as a potential platform for topical drug delivery. J Biomed Nanotechnol. 2012;8:280-9.

46. Manaia EB, Kaminski RCK, Soares CP, Meneau F, Pulcinelli $\mathrm{SH}$, Santilli CV, et al. Liquid crystalline formulations containing modified surface $\mathrm{TiO} 2$ nanoparticles obtained by sol-gel process. J Sol-Gel Sci Technol. 2012;63:251-7.

47. Beaucage G, Ulibarri TA, Black EP, Schaefer DW. Multiple Size Scale Structures in Silica-Siloxane Composites Studied by
Small-Angle Scattering. In: Mark JE, Lee C Y-C, Bianconi PA, editors. Hybrid Organic-Inorganic Composites. ACS Symposium Series; 1995. p.97-111.

48. Efrat R, Aserin A, Kesselman E, Danino D, Wachtel EJ, Garti N. Liquid micellar discontinuous cubic mesophase from ternary monoolein/ethanol/water mixtures. Colloids Surf A Physicochem Eng Asp. 2007;299:133-45.

49. Oyafuso MH, Carvalho FC, Chiavacci LA, Gremião MPD, Chorilli M. Design and characterization of silicone and surfactant based systems for topical drug delivery. J Nanosci Nanotechnol. 2015;15:817-26.

50. Cho BK, Jain A, Gruner SM, Wiesner U. Mesophase structuremechanical and ionic transport correlations in extended amphiphilic dendrons. Science. 2004;305:1598-601.

51. Schramm G. A practical approach to rheology and rheometry. 2nd ed. Karlsruhe: Haake; 1994.

52. Cid YP, Pedrazzi V, de Sousa VP, Pierre MBR. In vitro characterization of chitosan gels for buccal delivery of celecoxib: influence of a penetration enhancer. AAPS PharmSciTech. 2012;13:101-11.

53. Graciano TB, Coutinho TS, Cressoni CB, de Paula Freitas C, Pierre MBR, de Lima Pereira SA, et al. Using chitosan gels as a toluidine blue $\mathrm{O}$ delivery system for photodynamic therapy of buccal cancer: in vitro and in vivo studies. Photodiagn Photodyn Ther. 2015;12:98-107.

54. Perioli L, Pagano C, Mazzitelli S, Rossi C, Nastruzzi C. Rheological and functional characterization of new antiinflammatory delivery systems designed for buccal administration. Int J Pharm. 2008;356:19-28.

55. Calixto G, Yoshii AC, Rocha e Silva H, Stringhetti Ferreira Cury B, Chorilli M. Polyacrylic acid polymers hydrogels intended to topical drug delivery: preparation and characterization. Pharm Dev Technol. 2015;20:490-6.

56. Fonseca-Santos B, dos Santos AM, Rodero CF, Gremião MPD, Chorilli M. Design, characterization, and biological evaluation of curcumin-loaded surfactant-based systems for topical drug delivery. Int J Nanomedicine. 2016;11:4553.

57. Yaprak (Hızarcioğlu) Karavana S, Güneri P, Ertan G. Benzydamine hydrochloride buccal bioadhesive gels designed for oral ulcers: preparation, rheological, textural, mucoadhesive and release properties. Pharm Dev Technol. 2009;14:623-31.

58. Jones DS, Bruschi ML, de Freitas O, Gremião MPD, Lara EHG, Andrews GP. Rheological, mechanical and mucoadhesive properties of thermoresponsive, bioadhesive binary mixtures composed of poloxamer 407 and Carbopol@ 974P designed as platforms for implantable drug delivery systems for use in the oral cavity. Int J Pharm. 2009;373:49-58.

59. Şenyiğit T, Tekmen I, Sönmez Ü, Santi P, Özer Ö. Deoxycholate hydrogels of betamethasone-17-valerate intended for topical use: in vitro and in vivo evaluation. Int J Pharm. 2011;403:123-9.

60. Jones DS, Woolfson AD, Brown AF. Textural, viscoelastic and mucoadhesive properties of pharmaceutical gels composed of cellulose polymers. Int J Pharm. 1997;151:223-33.

61. Merino-Bohórquez V, Casas M, Caracuel F, Cameán M, Fernández-Anguita MJ, Ramírez-Soto G, et al. Physicochemical stability of a new topical timolol $0.5 \%$ gel formulation for the treatment of infant hemangioma. Pharm Dev Technol. 2015;20:562-9.

62. Repka MA, Mcginity JW. Physical-mechanical, moisture absorption and bioadhesive properties of hydroxypropylcellulose hot-melt extruded films. Biomaterials. 2000;21:1509-17.

63. Storpirtis S, Rodrigues D. In vitro evaluation of dissolution properties and degradation products of omeprazole in entericcoated pellets. Drug Dev Ind Pharm. 1998;24(11):1101-7.

64. Santos GSD, Pereira GG, Bender EA, Colomé LM, Guterres SS, Carvalho DCMD, et al. Development and characterization of lipid nanoparticles for dapsone topical application. Quim Nova. 2012;35:1388-94.

65. Silva C, Ribeiro A, Ferreira D, Veiga F. Administração oral de peptídios e proteínas: I. Estratégias gerais para aumento da biodisponibilidade oral. Braz J Pharm Sci. 2002;38:125-40.

66. Burke PA. Controlled release protein therapeutics: effects of process and formulation on stability. In: Wise DL, editor. Handbook of pharmaceutical controlled release technology. New York: Marcel-Dekker; 2000. p. 661-92. 
67. Zhou XH, Li Wan Po A. Peptide and protein drugs: I. Therapeutic applications, absorption and parenteral administration. Int J Pharm. 1991;75:97-115.

68. de Oliveira Cardoso VM, Cury BSF, Evangelista RC, Gremião MPD. Development and characterization of cross-linked gellan gum and retrograded starch blend hydrogels for drug delivery applications. J Mech Behav Biomed Mater. 2017;65:317-33.
69. Costa P, Lobo JMS. Modeling and comparison of dissolution profiles. Eur J Pharm Sci. 2001;13(2):123-33.

70. Papadopoulou V, Kosmidis K, Vlachou M, Macheras P. On the use of the Weibull function for the discernment of drug release mechanisms. Int J Pharm. 2006;309:44-50.

71. Dahl JE, Frangou-Polyzois MJ, Polyzois GL. In vitro biocompatibility of denture relining materials. Gerodontology. 2006;23:17-22. 